\title{
A Wave Based Method to predict the absorption, reflection and transmission coefficient of two-dimensional rigid frame porous structures with periodic inclusions
}

\author{
Elke Deckers ${ }^{\mathrm{a}, *}$, Claus Claeys $^{\mathrm{a}}$, Onur Atak ${ }^{\mathrm{a}}$, Jean-Philippe Groby ${ }^{\mathrm{b}}$, Olivier Dazel $^{\mathrm{b}}$, Wim Desmet $^{\mathrm{a}}$ \\ ${ }^{a}$ Department of Mechanical Engineering, Katholieke Universiteit Leuven, 3001 Heverlee, Belgium \\ ${ }^{b}$ Laboratiore d'Acoustique de l'Universiteé du Maine, L'Université Nantes Angers Le Mans, \\ Université du Maine, CNRS, UMR-6613 CNRS, Avenue Olivier Messiaen, 72085 Le Mans, France
}

\begin{abstract}
This paper presents an extension to the Wave Based Method to predict the absorption, reflection and transmission coefficients of a porous material with an embedded periodic set of inclusions. The porous unit cell is described using the Multi-Level methodology and by embedding Bloch-Floquet periodicity conditions in the weighted residual scheme. The dynamic pressure field in the semi-infinite acoustic domains is approximated using a novel wave function set that fulfills the Helmholtz equation, the Bloch-Floquet periodicity conditions and the Sommerfeld radiation condition. The method is meshless and computationally efficient, which makes it well suited for optimisation studies.
\end{abstract}

Keywords: acoustics, equivalent fluid, periodic structures, Wave Based Method, absorption, transmission

\section{Introduction}

In many noise control applications poroelastic materials are applied given their good sound absorption properties in the mid and high frequency range. As a rule of thumb, the thickness of the material should at least be around a quarter of the wavelength of the waves inside the medium to provide good absorption when backed by a rigid wall. This leads to bulky solutions in the low frequency range.

A common approach to improve low frequency absorption and sound transmission loss is to use multilayered structures consisting of different layers of porous, air and (visco-)elastic layers, e.g. [1, 2]. To this extent, also the anisotropy of poroelastic structures can be exploited [3].

Several other ways to improve the low-frequency absorption of porous materials have been proposed, using volume or surface heterogeneities. The first class of materials comprises double porosity materials $[4,5]$ and porous inclusions $[6,7]$, which act on pressure diffusion effects. Homogenisation theories are applied to come up with a valid, equivalent model. Besides, rigid periodic inclusions of different shapes [8,9] and embedded resonators $[10,11,12]$ have been

${ }^{*}$ Corresponding author, Tel.:+32 163726 79; Fax: +32 163229 87; http://www.mech.kuleuven.be/ E-mail address: Elke.Deckers@ mech.kuleuven.be.

Email address: Elke.Deckers@mech.kuleuven. be (Elke Deckers) 
studied, acting on trapped, modified, and local modes. In the second class, for instance, periodic irregular gratings can be used [13].

This papers focuses on a novel modelling concept for periodic inclusions in a two-dimensional porous matrix. The porous material is modelled as a rigid frame equivalent fluid, described by the so-called Johnson-Champoux-Allard (JCA) model $[14,15,16]$. Due to the rigid nature of the solid phase, only one wave type can propagate in this material and the governing physics are captured by a single Helmholtz equation. The applied complex bulk modulus and density account for the thermal and viscous losses in the material.

Different strategies are available in literature to study this type of problems. The Transfer Matrix Method [16] is well suited to predict absorption and transmission coefficients of laterally infinite layered structures, however, does not allow for periodic inclusions. For two-dimensional structures, a multipole method (MPM) can be used [7], directly exploiting the periodicity condition in the approximation of the dynamic fields. Its main drawback is that it only allows for circular inclusions. Besides, a mode matching approach can be used, based on a rectangular decomposition of the problem domain [17]. Periodicity conditions can also be embedded in the framework of the Finite Element Method, allowing to model any inclusion shape. The infinite, acoustic part(s) of the problem can be modelled by using appropriate absorbing boundary conditions or by a modal (Bloch-Floquet) expansion [9].

This paper presents an extension to the Wave Based Method (WBM), which allows to evaluate the effect of periodic inclusions on acoustic quantities of interest such as absorption and transmission coefficients. The WBM $[18,19]$ is based on an indirect Trefftz approach [20]; it uses a set of exact solutions of the governing differential equations, so-called wave functions, to approximate the field variables. Boundary and interface residuals are minimised in an integral sense using a weighted Galerkin approach. The resulting system of equations can be solved for the contribution factors of all individual wave functions. It has been shown that a sufficient condition for the method to converge is the convexity of the problem domain [18]. Non-convex domains have to be partitioned into convex subdomains. The WBM has been applied to (un)bounded acoustic [21, 22], plate bending [23, 24] and plate membrane problems [25], poroelastic [26, 27] and general vibro-acoustic problems [28] and its computational efficiency as compared to standard Finite Element and Boundary Element methods has been illustrated for many verification examples.

For problems containing (multiple) inclusions, however, the convexity requirement may lead to a very large number of subdomains, jeopardising the efficiency of the WBM. When considering circular inclusions, it is even impossible to obtain convex subdomains. The Multi-Level framework [29] presents an elegant concept to account for multiple scattering problems in unbounded problem domains: each scatterer is considered in a separate 'level'. In every level, the scattering due to a single object is modelled with the existing WBM for unbounded problems, as if the other scatterers do not exist. By applying the superposition principle and linking the pressure fields of all levels together via a weighted residual approach, a fully coupled numerical model is obtained. A similar reasoning can be made for bounded domains with (multiple) inclusions [30] i. e. inclusions and bounded domains are modelled as if they are separate entities and they are coupled through a weighted residual formulation to form a fully coupled model. This approach allows to handle inclusions without violating the convexity of the bounded domains. 
To be applicable for unit cell models for acoustic absorption and transmission cases, the Multi-Level WBM (MLWBM) [30] is extended in this paper to account for periodicity conditions. This allows to model only a unit cell of the problem at hand to obtain the response fields within the problem domain. For the bounded, porous part of the problem, periodicity conditions are formulated and included in the weighted residual scheme. For the unbounded acoustic part(s) of the problem, a novel wave function set is presented such that the Helmholtz equation, the Sommerfeld radiation condition and the periodicity conditions are automatically fulfilled. As compared to standard Finite Element procedures, this novel method offers some advantages; it is computationally efficient and unbounded domains can inherently be accounted for. Besides, as the procedure is meshless, optimisation studies can easily be implemented. As compared to the MPM, the WBM is not restricted to inclusions with analytical solutions.

The paper is organised as follows. Section 2 discusses the general problem description. Section 3 briefly recalls the WBM and the Multi-Level approach. Section 4 extends the WBM for absorption and transmission calculations using unit cell models. Bloch-Floquet boundary conditions are applied to the porous part of the problem and the resulting residuals are minimised using a weighted Galerkin approach. Novel wave functions are introduced for the periodic, semi-unbounded acoustic domain(s) of interest. Section 5 demonstrates the method with four numerical examples. The first one shows the convergence of the method with respect to the analytical solution of a simple problem, next, an absorption case available in literature and a transmission case are studied and the obtained results are validated using the MPM. The fourth application case considers an optimisation study. Finally, Section 6 summarises the main conclusions of the paper.

\section{Problem description}

This section briefly describes the mathematical problem setting used for a general 2D periodic coupled (semi-) infinite acoustic-porous problem containing rigid inclusions, as shown in Figure 1. A time-harmonic motion with $e^{\mathrm{j} \omega t}$-dependence is assumed, where $\mathrm{j}$ is the imaginary unit, $\mathrm{j}^{2}=-1, \omega$ is the circular frequency and $t$ is the time.

The total problem domain $\Omega$ can be divided into two non-overlapping domains constituted of different physical media: $\Omega_{a}$ and $\Omega_{e}$, containing air and a porous medium, described as an equivalent fluid, respectively. The periodic porous structure is excited by an impinging plane wave with amplitude $A$ and wave number $\mathbf{k}$, incident at an angle $\theta$, measured counterclockwise from the positive $x$-axis. The thickness of the porous structure is denoted by $L_{y}$ and the heterogeneities are periodic in the $x$-direction with period $L_{x}$.

In the application cases of this paper, the inclusions are considered rigid (i.e. Neumann boundary conditions). In a completely similar way, other boundary conditions like Dirichlet and Robin boundary conditions, or the coupling between different equivalent fluids can be introduced, as described in [31].

It is assumed that the porous material has a rigid frame and can be modelled, using homogenisation techniques, as an equivalent fluid medium $[16,31]$, with a complex and frequency dependent effective density $\rho_{e}$ and effective bulk modulus $K_{e}$. The Johnson-Champoux-Allard model [16] is used to represent the dynamic behaviour of the equivalent 


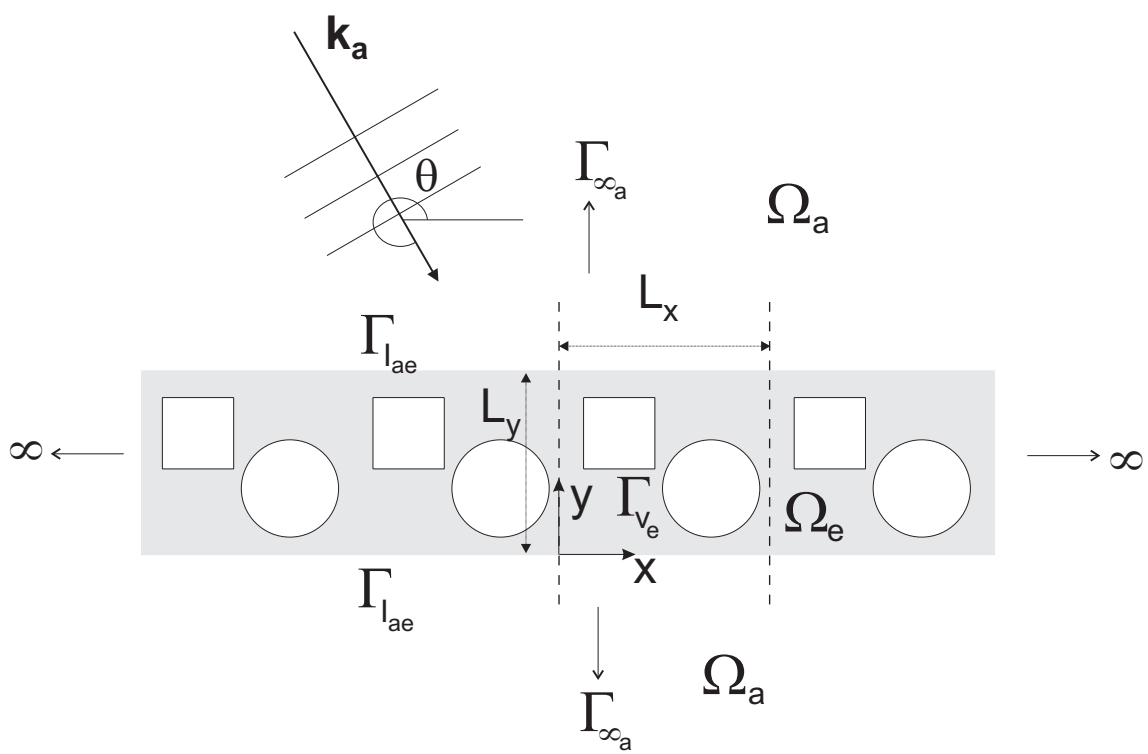

Figure 1: General problem description of a 2D infinite porous structure with periodic inclusions, coupled to two infinite acoustic domains.

fluid and is detailed in Appendix A. The steady-state pressure $p_{e}(\mathbf{r})$, inside the medium $\Omega_{e}$, is governed by the Helmholtz equation.

Under the assumption of an inviscid fluid with ambient fluid density $\rho_{a}$ and speed of sound $c_{a}$, a linear system and an adiabatic process, also the acoustic pressure, $p_{a}(\mathbf{r})$, in medium $\Omega_{a}$ is governed by the inhomogeneous Helmholtz equation. Consequently, the steady state pressure $p_{\bullet}(\mathbf{r})$, with $\bullet=a$ for the acoustic and $\bullet=e$ for the equivalent fluid case is given by:

$$
\mathbf{r} \in \Omega_{\bullet}: \nabla^{2} p_{\bullet}(\mathbf{r})+k_{\bullet}^{2} p_{\bullet}(\mathbf{r})=\mathcal{F}_{\bullet}(\mathbf{r}),
$$

where $\nabla^{2}$ is the Laplacian operator and $k_{\bullet}=\frac{\omega}{c_{\bullet}}=\omega$ is the wave number of medium $\Omega_{\bullet}$. The fluid is excited by a source defined by $\mathcal{F}_{\bullet}(\mathbf{r})$. In the remainder of the paper the subscript $\bullet$ can be replaced by $a$ if acoustic quantities are considered or $e$ when equivalent fluid quantities are considered.

Due to the geometrical periodicity and the plane wave nature of the excitation, the resulting dynamic fields have to be periodic in the $x$-direction. It is thus sufficient to determine the dynamic field in a single reference cell and use the Bloch-Floquet relation to obtain the dynamic fields in all other cells [32, 33]:

$$
p_{\bullet}\left(x+N L_{x}, y\right)=p_{\bullet}(x, y) e^{-\mathrm{j} k_{a x} N L_{x}}, \forall N \in \mathbb{Z},
$$

where $k_{a x}=k_{a} \cos \theta$ and $N$ is an integer which indicates the position of the cell of interest relative to the reference cell.

Since the Helmholtz equation is a second order differential equation, one boundary condition needs to be specified at each point of the boundary of the subdomains in order to obtain a well-posed problem. The boundary of medium $\Omega$. is denoted as $\Gamma_{\bullet}=\partial \Omega_{\bullet}$. The acoustic boundary $\Gamma_{a}$ consists of two separate parts: the exterior boundary at infinity, $\Gamma_{\infty_{a}}$, 
and the coupling interfaces with the equivalent fluid domain, $\Gamma_{I_{a e}}$. On the former the Sommerfeld radiation condition applies, ensuring that no energy is reflected at infinity [34], leading to the following residual:

$$
\mathbf{r} \in \Gamma_{\infty_{\bullet}}: R_{\infty_{\bullet}}(\mathbf{r})=\lim _{|\mathbf{r}| \rightarrow \infty}\left(\left(\frac{\partial p_{\bullet}(\mathbf{r})}{\partial|\mathbf{r}|}+\mathrm{j} k_{\bullet} p_{\bullet}(\mathbf{r})\right)\right)=0 .
$$

The boundary of the considered equivalent fluid medium $\Omega_{e}$ also consists of two separate parts: the rigid boundaries determined by the inclusions, $\Gamma_{v_{e}}$, and the coupling interfaces with the acoustic subdomains $\Gamma_{I_{a e}}$. On the former, the Neumann residual applies:

$$
\mathbf{r} \in \Gamma_{v_{\bullet}}: R_{v_{\bullet}}(\mathbf{r})=\mathcal{L}_{v_{\bullet}}\left(p_{\bullet}(\mathbf{r})\right)-\bar{v}_{\bullet}, n=0
$$

with $\bar{v}_{\bullet, n}$ the prescribed value for the normal velocity, being $0 \mathrm{~m} / \mathrm{s}$ for a rigid boundary. The velocity operator is defined as:

$$
\mathcal{L}_{v_{\bullet}}\left(p_{\bullet}(\mathbf{r})\right)=\frac{\mathrm{j}}{\rho_{\bullet} \omega} \frac{\partial p_{\bullet}(\mathbf{r})}{\partial \gamma_{\mathbf{n}}},
$$

with $\gamma_{\mathbf{n}}$ the normal direction to the boundary, pointing outwards.

On the interfaces $\Gamma_{I_{a e}}$ between the acoustic and the porous medium, the continuity of pressure and velocity are imposed using the following residuals:

$$
\mathbf{r} \in \Gamma_{I_{\bullet \star}}:\left\{\begin{array}{l}
R_{I p_{\bullet \star}}(\mathbf{r})=p_{\bullet}(\mathbf{r})-p_{\star}(\mathbf{r})=0, \\
R_{I v_{\bullet \star}}(\mathbf{r})=\mathcal{L}_{v_{\bullet}}\left(p_{\bullet}(\mathbf{r})\right)+\mathcal{L}_{v_{\star}}\left(p_{\star}(\mathbf{r})\right)=0,
\end{array}\right.
$$

where both $\bullet$ and $\star$ can be $a$ or $e$. The governing Helmholtz equations in the different domains (1), the periodicity conditions (2), the applied boundary conditions (3)-(4) and interface conditions (6) define a unique pressure field $p_{\bullet}(\mathbf{r})$.

\section{Wave Based Method}

The WBM [18] is a numerical prediction technique which is based on an indirect Trefftz approach [20]. It approximates the field variables by a weighted sum of wave functions which inherently satisfy the governing equation, in this case the Helmholtz equation (1). This section introduces the theory and notations needed as a starting point for the WB unit cell model development, presented in Section 4. Section 3.1 briefly reviews the basic modelling steps for 2D bounded and unbounded Helmholtz problems. Section 3.2 recalls the Multi-Level concept.

\subsection{WBM for 2D Helmholtz problems}

The general modelling procedure consists of four steps, which will be briefly discussed afterwards:

A. Partitioning of the problem domain into convex subdomains,

B. Selection of the wave functions in the pressure expansion within each subdomain,

C. Construction of the system of equations via a weighted residual formulation of the boundary conditions and the continuity conditions,

D. Solution of the system of equations and postprocessing of the dynamic variables.

A more detailed description can be found in literature, e.g. [18, 19, 21, 22, 35]. 


\subsubsection{Partitioning into subdomains}

A sufficient condition for the WBM to converge is that the considered subdomains are convex [18]. Non-convex problem domains have to partitioned into a number of convex subdomains. In the case of unbounded problems, an initial partitioning of the unbounded domain into a bounded and an unbounded region by a truncation curve $\Gamma_{t_{0}}$ with radius $R_{t_{\bullet}}$ precedes the partitioning into convex subdomains [36]. Figure 2 illustrates the principle. The unbounded region exterior to $\Gamma_{t_{\bullet}}$ constitutes one subdomain.

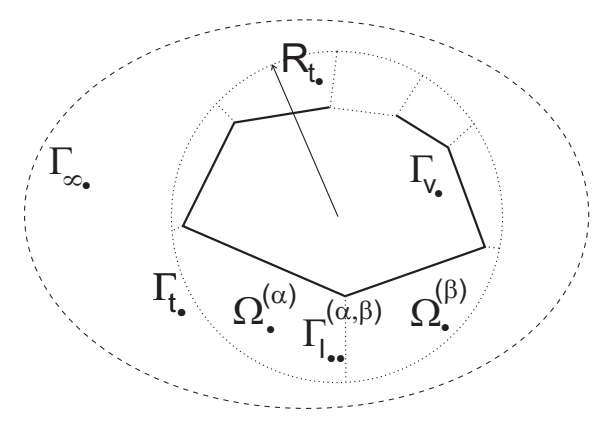

Figure 2: WB partitioning of the 2D unbounded Helmholtz problem. Subdomain interfaces are shown in dotted line ( $\cdots)$. For sake of simplicity only Neumann boundary conditions are indicated on the bounded part of the problem boundary.

The problem domain $\Omega_{\bullet}$ is thus subdivided into $N_{\Omega}$ non-overlapping subdomains $\Omega_{\bullet}^{(\alpha)}\left(\alpha=1, \ldots, N_{\Omega_{\bullet}}\right)$, which may be bounded or unbounded. On the interface $\Gamma_{I_{.}}^{(\alpha, \beta)}$ between two subdomains $\Omega_{\bullet}^{(\alpha)}$ and $\Omega_{\bullet}^{(\beta)}$, containing the same medium -, continuity conditions need to be imposed. Different coupling conditions can be considered. In this manuscript impedance continuity conditions are applied and are given in Appendix B.

\subsubsection{Pressure expansion in each subdomain $\Omega_{\bullet}^{(\alpha)}$}

The steady-state pressure field $p_{\bullet}^{(\alpha)}(\mathbf{r})$ in subdomain $\Omega_{\bullet}^{(\alpha)}$ is approximated by a solution expansion $\hat{p}_{\bullet}^{(\alpha)}(\mathbf{r})$ :

$$
p_{\bullet}^{(\alpha)}(\mathbf{r}) \simeq \hat{p}_{\bullet}^{(\alpha)}(\mathbf{r})=\sum_{w=1}^{n_{\bullet}^{(\alpha)}} p_{\bullet, w}^{(\alpha)} \Phi_{\bullet, w}^{(\alpha)}(\mathbf{r})+\hat{p}_{\bullet, q}(\mathbf{r})=\boldsymbol{\Phi}_{\bullet}^{(\alpha)}(\mathbf{r}) \mathbf{p}_{\bullet, w}^{(\alpha)}+\hat{p}_{\bullet, q}(\mathbf{r}) .
$$

The wave function contributions $p_{\boldsymbol{\bullet}, w}^{(\alpha)}$ are the weighting factors for each of the selected wave functions $\Phi_{\boldsymbol{\bullet}, w}^{(\alpha)}$. All weighting factors together form the vector of degrees of freedom $\mathbf{p}_{\bullet, w}^{(\alpha)}$. The corresponding a priori defined wave functions are collected in the row vector $\boldsymbol{\Phi}_{\bullet}^{(\alpha)} . \hat{p}_{\bullet}, q(\mathbf{r})$ represents a particular solution resulting from source terms in the right hand side of the inhomogeneous Helmholtz equation (1). The set of all $n_{\bullet}, W=\sum_{\alpha=1}^{N_{\Omega}} n_{\bullet, w}^{(\alpha)}$ wave function contributions $p_{\bullet, w}$ is collected in the column vector $\mathbf{p}_{\bullet, w}$, while the row vector $\boldsymbol{\Phi}_{\boldsymbol{\bullet}}$ contains all $n_{\bullet}, W$ wave functions. Expressions for the selected wave functions for $2 \mathrm{D}$ bounded and unbounded domains are detailed in Appendix $\mathrm{C}$.

\subsubsection{Construction of the system of equations}

The proposed wave functions (C.2) and (C.4) exactly satisfy the Helmholtz equation (1) inside the domain $\Omega$. and the wave functions for unbounded domains also fulfil the Sommerfeld radiation condition (3) at the boundary at 
infinity, $\Gamma_{\infty}$. Consequently only the boundary conditions and subdomain continuity conditions have to be enforced. This is done via a weighted residual formulation..

For each subdomain, the boundary and interface residuals are orthogonalised with respect to a weighting function $\tilde{p}_{\bullet}^{(\alpha)}(\mathbf{r})$ or its derivative. Like in the Galerkin weighting procedure, used in the FEM, the weighting functions are expressed in terms of the same sets of wave functions used in the pressure expansions of the field variables (7):

$$
\tilde{p}_{\bullet}^{(\alpha)}(\mathbf{r})=\sum_{w=1}^{n_{\bullet, w}^{(\alpha)}} \tilde{p}_{\bullet, w}^{(\alpha)} \Phi_{\bullet, w}^{(\alpha)}(\mathbf{r})=\boldsymbol{\Phi}_{\bullet}^{(\alpha)}(\mathbf{r}) \tilde{\mathbf{p}}_{\bullet, \mathbf{w}}^{(\alpha)} .
$$

Substitution of the field variable expansions and the weighting function expansions in the weighted residual formulation, leads to an algebraic equation which links together the wave function contribution factors of the considered subdomain $\Omega_{\bullet}^{(\alpha)}$ and those of the adjacent subdomains $\Omega_{\bullet}^{(\beta)}$. This procedure is repeated for each subdomain. Enforcing that these equations should hold for any weighting function and thus for all possible combinations of $\tilde{p}_{\bullet, w}^{(\alpha)}$ results in frequency dependent system of equations:

$$
\left[\begin{array}{cccc}
\mathbf{A}_{\bullet}^{(1)} & \mathbf{C}_{\bullet \bullet}^{(1,2)} & \cdots & \mathbf{C}_{\bullet \bullet}^{\left(1, N_{\Omega_{\bullet}}\right)} \\
\mathbf{C}_{\bullet \bullet}^{(2,1)} & \mathbf{A}_{\bullet}^{(2)} & \cdots & \mathbf{C}_{\bullet}^{\left(2, N_{\Omega_{\bullet}}\right)} \\
\vdots & \vdots & \ddots & \vdots \\
\mathbf{C}_{\bullet}^{\left(\mathrm{N}_{\left.\Omega_{0}, 1\right)}\right)} & \mathbf{C}_{\bullet}^{\left(\mathrm{N}_{\left.\Omega_{\bullet}, 2\right)}\right.} & \cdots & \mathbf{A}_{\bullet}^{\left(\mathrm{N}_{\Omega_{\bullet}}\right)}
\end{array}\right] \cdot\left[\begin{array}{c}
\mathbf{p}_{\bullet, w}^{(1)} \\
\mathbf{p}_{\bullet, w}^{(2)} \\
\vdots \\
\mathbf{p}_{\bullet, w}^{\left(\mathrm{N}_{\Omega_{\bullet}}\right)}
\end{array}\right]=\left[\begin{array}{c}
\mathbf{f}_{\bullet}^{(1)} \\
\mathbf{f}_{\bullet}^{(2)} \\
\vdots \\
\sum_{\beta} \mathbf{f}_{\bullet}^{\left(\mathrm{N}_{\Omega_{\bullet}}\right)}
\end{array}\right] .
$$

The matrices $\mathbf{A}_{\bullet}^{(\alpha)}$ are the system matrices, the matrices $\mathbf{C}_{\bullet \bullet}^{(\alpha, \beta)}$ are the coupling matrices, being nonzero for two adjacent subdomains $\Omega_{\bullet}^{(\alpha)}$ and $\Omega_{\bullet}^{(\beta)}$, and $\mathbf{f}_{\bullet}$ are loading vectors resulting from non-homogeneous boundary conditions and source contributions. General expressions of the weighted residual formulation and the resulting system matrices can be found in literature $[18,36,37]$.

\subsubsection{Solution and postprocessing}

The resulting model (9) can be solved for the unknown wave function contributions $\mathbf{p}_{\bullet}, \mathbf{w}$. The final step in the modelling process is back substitution of these contribution factors into the pressure expansions (7), using wave function sets (C.2) or (C.4), yielding an analytical description of the approximated dynamic pressure field $\hat{p}$ •(r). Also derived quantities such as velocities or intensities can be obtained.

\subsection{Multi-Level approach}

The Multi-Level approach was, in a first step, developed for unbounded acoustic multiple scattering configurations [29]. The main idea for unbounded scattering problems is to consider each of the scatterers in the problem as a different 'level' of the problem. In every level, the scattering of one specific scatterer is described, using the unbounded WBM, as detailed in Section 3.1. The total solution field, in the region exterior to the truncation circles of all scatterers is called the compound subdomain. The pressure field within this compound subdomain is then written as a weighted sum of unbounded wave functions coming from all unbounded levels, and is called the compound wave function set. The existence and uniqueness of this composition is proven by Grote [38]. The different wave fields resulting from 
the different levels are thus combined and coupled via the weighted residual approach minimising the boundary and interface residuals. The resulting WB system model is fully coupled and is solved at once.

Afterwards, the WBM Multi-level approach was extended to bounded problems [30]. As compared to the MultiLevel approach for unbounded problems, one additional level is created, considering the bounded part of the problem as if no inclusions are present. The other levels consider again the scattering of each of the inclusions seperately as if the bounded domain and the other inclusions are not present. The total solution field can then be obtained by combining the wave functions resulting from the different levels together and by minimising the error on all boundaries and interfaces using the weighted residual approach. The concept is illustrated in Figure 3 showing a bounded problem domain with two inclusions.

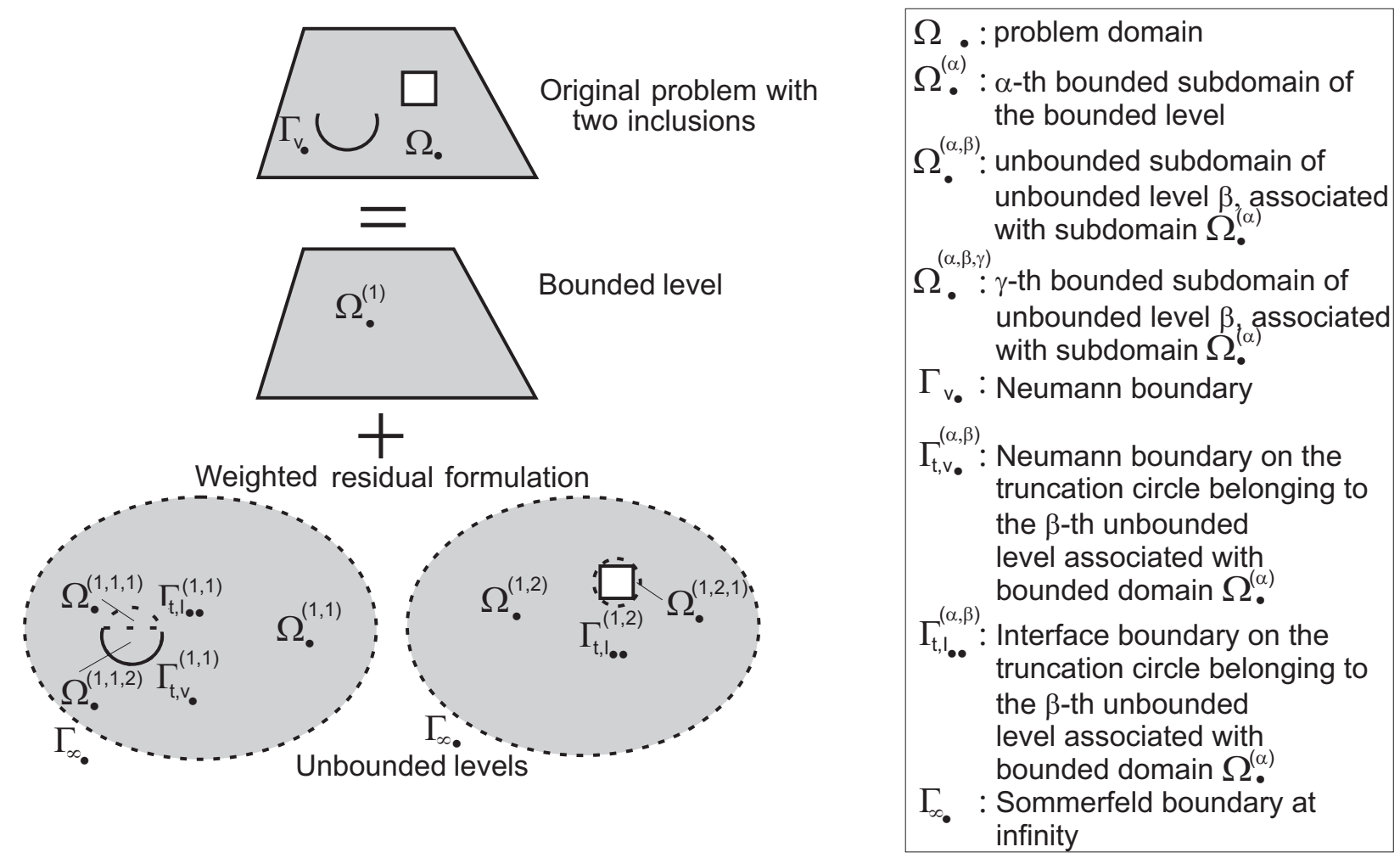

Figure 3: Graphical representation of the Multi-Level modelling concept for bounded problems.

The Multi-Level WBM approach consists of four steps that are briefly revisited. A detailed discussion can be found in [30].

\section{A. Division of the original problem into levels and, if required, into subdomains:}

In a first step the original problem is divided into a number of levels: the first, 'bounded', level includes the bounded problem as if there were no inclusions present, see Figure 3. If needed, this bounded domain can be partitioned into convex subdomains (not needed in Figure 3). These bounded subdomains of the bounded level are indicated $\Omega_{\bullet}^{(\alpha)}$ where $\alpha$ refers to the index of the bounded subdomain. 
The other 'unbounded' levels consider the scattering of one single inclusion as if the bounded domain and the other inclusions are not present. Each of the scatterers, belonging to a certain unbounded level is physically located in a bounded subdomain $\Omega_{\bullet}^{(\alpha)}$ of the bounded level. Each subdomain $\Omega_{\bullet}^{(\alpha)}$ may contain $N_{u b}^{(\alpha)}$ inclusions. Following the WBM for unbounded problems, the unbounded problem domains of the unbounded levels are first partitioned into an unbounded and a bounded region. The truncation circles are denoted by $\Gamma_{t, *}^{(\alpha, \beta)}$ where $\alpha$ refers to the index of the bounded subdomain of the bounded level in which the inclusion is located and $\beta$ indicates the index of the unbounded level. The truncation circle $\Gamma_{t, *}^{(\alpha, \beta)}$ is subdivided into different zones, indicated with subscript $*$, on which different boundary or interface conditions are imposed. In this manuscript only Neumann $\left(*=v_{\bullet}\right)$ boundary conditions or continuity conditions between the resulting unbounded and bounded domains outside and inside the truncation $\left(*=I_{\bullet \bullet}\right)$ are considered $: \Gamma_{t, *}^{(\alpha, \beta)}=\Gamma_{t, v_{\bullet}}^{(\alpha, \beta)} \cup \Gamma_{t, I_{\bullet \bullet}}^{(\alpha, \beta)}$. The unbounded acoustic region exterior to $\Gamma_{t, \bullet}^{(\alpha, \beta)}$ is denoted $\Omega_{\bullet}^{(\alpha, \beta)}$, with $\alpha$ and $\beta$ as defined above. The bounded regions inside the truncation circles are partitioned into $N_{b}^{(\alpha, \beta)}$ convex subdomains, indicated by dashed lines. The resulting bounded subdomains inside the truncation circles are indicated $\Omega_{\bullet}^{(\alpha, \beta, \gamma)}$ with $\alpha$ and $\beta$ as defined above and $\gamma$ the index of the bounded subdomain within unbounded level $\beta$.

The problem depicted in Figure 3 is thus divided into three levels: the bounded level results in a single bounded subdomain, the two unbounded levels are partitioned into one unbounded and two and four bounded subdomains, respectively.

\section{B. Selection of wave functions for the different levels:}

For each subdomain belonging to a level, a suitable wave function set is selected to describe its dynamic field variables (7). The selection of wave functions for bounded and unbounded subdomains is discussed in Appendix C. Once the field variables are defined for each subdomain, they are combined using the superposition principle. Let us denote $\hat{p}_{\bullet}^{(\alpha)}(\mathbf{r})$ the field variable of the $\alpha$-th bounded subdomain $\Omega_{\bullet}^{(\alpha)}$ and $\hat{p}_{\bullet}^{(\alpha, \beta)}(\mathbf{r})$ the field variable of the $\beta$-th unbounded subdomain $\Omega_{\bullet}^{(\alpha, \beta)}$ of which the inclusion lies in bounded domain $\Omega_{\bullet}^{(\alpha)}$, with $\beta=1, \ldots, N_{u b}^{(\alpha)}$. Then the compound pressure field $\hat{p}_{\bullet}^{\left(\alpha^{\prime}\right)}(\mathbf{r})$ in the compound subdomain $\Omega_{\bullet}^{\left(\alpha^{\prime}\right)}=\Omega_{\bullet}^{(\alpha)} \cap \Omega_{\bullet}^{(\alpha, 1)} \cap \ldots \cap \Omega_{\bullet}^{\left(\alpha, N_{u b}^{(\alpha)}\right)}$ can be written as [30]:

$$
\mathbf{r} \in \Omega_{\bullet}^{\left(\alpha^{\prime}\right)}: \hat{p}_{\bullet}^{\left(\alpha^{\prime}\right)}(\mathbf{r})=\hat{p}_{\bullet}^{(\alpha)}(\mathbf{r})+\sum_{\beta=1}^{N_{u b}^{(\alpha)}} \hat{p}_{\bullet}^{(\alpha, \beta)}(\mathbf{r}) .
$$

For the considered Multi-Level problem, the resulting compound subdomain $\Omega^{\left(1^{\prime}\right)}$ is illustrated in Figure 4.

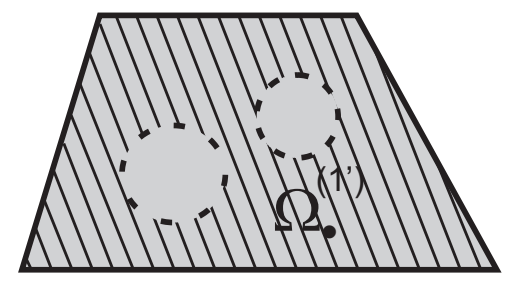

Figure 4: Representation of the compound subdomain $\Omega_{\bullet}^{\left(1^{\prime}\right)}$. 
The pressure field in the bounded domains $\Omega_{\bullet}^{(\alpha, \beta, \gamma)}$ within the unbounded levels is described using only regular bounded wave functions belonging to that particular subdomain, see Equation (C.2). Note that the total problem domain $\Omega_{\bullet}$ is uniquely defined by all subdomains $\Omega_{\bullet}^{\left(\alpha^{\prime}\right)}$ and $\Omega_{\bullet}^{(\alpha, \beta, \gamma)}$, so for each compound subdomain of the problem an appropriate field variable expansion is defined.

\section{Construction of the system of equations:}

The boundary and interface conditions need to be enforced, which is achieved by using a weighted Galerkin approach, using the compound wave function sets for subdomains $\Omega_{\bullet}^{\left(\alpha^{\prime}\right)}$ and regular bounded wave functions for the bounded subdomains $\Omega_{\bullet}^{(\alpha, \beta, \gamma)}$ to approximate the field variables within the residuals on the boundaries and interfaces.

The choice of test functions may be different for the different boundaries. In general, to have valid test functions, they need to be able to represent an arbitrary field on that specific boundary. As unbounded wave functions can accurately represent any field on the truncation surface $\Gamma_{t, *}^{(\alpha, \beta)}$ of the subdomain $\Omega_{\bullet}^{\left(\alpha^{\prime}\right)}$ they belong to, they are used as weighting functions on that truncation boundary. Similarly the bounded wave functions defined in $\Omega_{\bullet}^{(\alpha)}$ can be used as weighting functions on the boundaries defining the bounded level. The wave functions of $\Omega_{\bullet}^{(\alpha, \beta, \gamma)}$ are used as weighting functions on boundaries of that subdomain.

D. Solution and post-processing:

The system matrices are solved for the unknown contribution factors of all wave functions originating from all levels, at once. In a post-processing step, the response field can be evaluated using the field variable expansions of $\Omega_{\bullet}^{\left(\alpha^{\prime}\right)}$ and $\Omega_{\bullet}^{(\alpha, \beta, \gamma)}$.

A detailed description can be found in [30] for acoustic and plate membrane problems.

\section{Wave Based Method for periodic structures}

This section extends the existing WBM framework to account for periodic problem settings as shown in Figure 1. As pointed out in Section 2, due to the periodicity, it suffices to study the response in one unit cell to be able to reconstruct the response in any point of the system. The considered unit cell, with an indication of the subdomains and the boundaries, is given in Figure 5.

The bounded, porous part of the problem domain can be modelled using the Multi-Level WBM [30], together with the equivalent fluid properties to determine the wave numbers in the pressure expansions.

Besides regular coupling and boundary conditions, Bloch-Floquet relations have to be imposed on the pressure and velocity values on the periodic boundaries $\Gamma_{B F L_{e}}$ and $\Gamma_{B F R_{e}}$, such that the resulting dynamic fields are periodic. These conditions will be discussed in Section 4.1.

In the semi-unbouded periodic acoustic subdomains, the existing semi-unbounded wave functions [39] cannot be directly applied, since they do not inherently fulfil the Bloch-Floquet periodicity condition and would require an integration over infinitely long acoustic boundaries. Instead, a semi-unbounded wave function expansion is used 


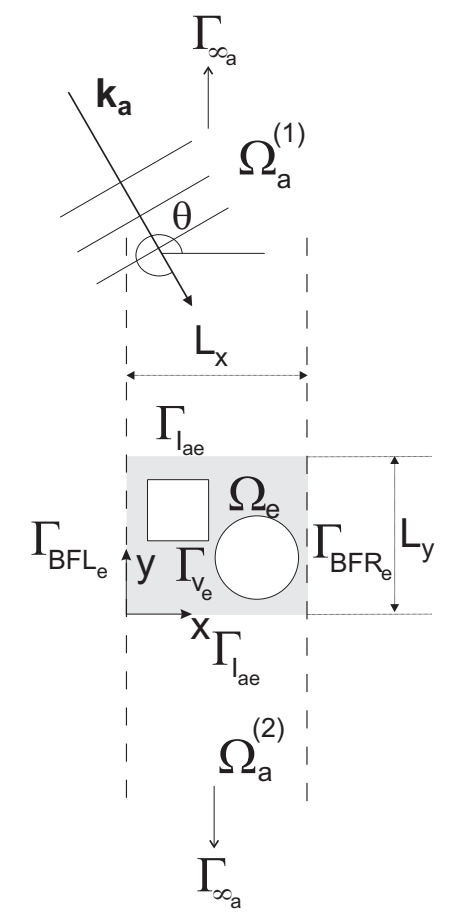

Figure 5: General problem setting of a unit cell of a periodic acoustic-porous problem with inclusions.

that inherently fulfils (i) the Bloch-Floquet condition, (ii) the Helmholtz equation and (iii) the Sommerfeld radiation condition. This wave function set is truncated such that its wave functions spatially match the wave functions applied in the bounded subdomain. This wave function set is discussed in Section 4.2.

The final subsection discusses the evaluation of the reflection, transmission and absorption coefficients.

\subsection{Bloch-Floquet boundary conditions in the Multi-Level WBM framework}

Contrary to the Multipole Method (MPM) [7], in which the pressure expansions do fulfil the Bloch-Floquet periodicity condition, that condition is weakly satisfied in the Multi-Level WBM framework. The advantage of using the WBM is, however, that there is no restriction to circular inclusions. Inclusions of moderate geometrical complexity can be dealt with using the regular Multi-Level WBM as is presented in this manuscript. When geometrically complex inclusions are considered, the recently developed hybrid Boundary Element-Wave Based Method [40] can be used. This methodology uses the concept of the Multi-Level method and accounts for the scattering of the geometrically complex inclusions by using the Boundary Element Method instead of the WBM for unbounded problems.

The Bloch-Floquet periodicity conditions have to be imposed on the left and right boundary of the porous unit cell. On the boundary $\Gamma_{B F L_{e}}$ the periodicity of pressure is explicitly imposed, whereas on the boundary $\Gamma_{B F R_{e}}$ the periodicity of the normal velocity is imposed. This leads to the following residuals, relating the dynamic fields on 
both boundaries:

$$
\begin{array}{ll}
\mathbf{r} \in \Gamma_{B F L_{e}}: & R_{B F L_{e}}(\mathbf{r})=p(\mathbf{r})-p\left(\mathbf{r}^{\mathbf{r}}\right) e^{\mathrm{j} k_{a x} L_{x}}=0, \\
\mathbf{r} \in \Gamma_{B F R_{e}}: & R_{B F R_{e}}(\mathbf{r})=\mathcal{L}_{v_{e}}(p(\mathbf{r}))+\mathcal{L}_{v_{e}}\left(p\left(\mathbf{r}^{\mathbf{l}}\right)\right) e^{-\mathrm{j} k_{a x} L_{x}}=0,
\end{array}
$$

in which $\mathbf{r}^{\mathbf{l}} \in \Gamma_{B F L_{e}}$ and $\mathbf{r}^{\mathbf{r}} \in \Gamma_{B F R_{e}}$. These residuals are minimised using the weighted residual approach, see Section 4.3 .

\subsection{Semi-unbounded Bloch-Floquet acoustic wave functions}

In order to avoid integrating residuals on infinite acoustic domains, the wave functions in the semi-unbounded acoustic domains are selected to fulfil not only the Helmholtz equation (1) and the Sommerfeld radiation condition (3), but also the Bloch-Floquet periodicity condition (2). The acoustic pressure in a semi-unbounded, periodic acoustic domains is also approximated using a weighted sum of wave functions (7). The wave functions $\Phi_{a, w}^{(\alpha)}(\mathbf{r})$ for a semiunbounded periodic domain are in this case based on a plane wave expansion:

$$
\Phi_{a, w}^{(\alpha)}(\mathbf{r}(x, y))=e^{-\mathrm{j}\left(k_{B F_{a, x w}}^{(\alpha)} x+k_{B F_{a, y w}}^{(\alpha)} y\right)} .
$$

The wave number components $k_{B F_{a, x w}}^{(\alpha)}$ are selected such that the periodicity condition (2) is fulfilled:

$$
k_{B F_{a, x w}}^{(\alpha)}=k_{a x}+\frac{2 w_{1}^{(\alpha)} \pi}{L_{x}},
$$

with $w_{1}^{(\alpha)}=\ldots,-2,-1,0,1,2, \ldots$. In order to fulfil the Helmholtz equation, the wave numbers $k_{B F_{y w}}^{(\alpha)}$ are selected as:

$$
k_{B F_{y w}}^{(\alpha)}= \pm
$$

and the sign of the root is selected such that the waves are purely outgoing and consequently the Sommerfeld condition is fulfilled. For the two acoustic domains $\Omega_{a}^{(1)}$ and $\Omega_{a}^{(2)}$, different signs are thus selected. The incoming plane wave is in domain $\Omega_{a}^{(1)}$ accounted for using the particular solution presented in equation (C.5).

The semi-unbounded Bloch-Floquet wave functions (13) are coupled to the Multi-Level expansion in the periodic porous domain on the interface $\Gamma_{I_{a e}}$ by minimisation of the residuals (6).

\subsection{Construction of the WB system of equations}

The WB system of equations is constructed using a weighted Galerkin approach, minimising the residuals on the boundaries and interfaces. As an illustration, a general transmission problem is considered. To clarify the approach, the different steps are also applied the unit cell of Figure 5.

Firstly, the porous part of the problem is divided into a number of levels. It is assumed that the first (bounded) level contains a convex domain $\Omega_{e}$ and no further partitioning into convex subdomains is required. Consequently this subdomain is denoted by $\Omega_{e}^{(1)}$. Considering infinite, periodic structures, this is a reasonable assumption, however, the expression below can be straightforwardly extended when this is not the case. It is assumed that $N_{u b}^{(1)}$ rigid inclusions are embedded in the unit cell, leading to $N_{u b}^{(1)}$ unbounded levels and a compound subdomain $\Omega_{e}^{\left(1^{\prime}\right)}=\Omega_{e}^{(1)} \cap \Omega_{e}^{(1,1)} \cap \ldots \cap \Omega_{e}^{\left(1, N_{u b}^{(1)}\right)}$. 
Each unbounded level $\beta$ contains $N_{b}^{(\alpha, \beta)}$ bounded convex subdomains $\Omega_{e}^{(\alpha, \beta, \gamma)}$. The porous part of the unit cell problem of Figure 5 is thus decomposed into three levels, shown in Figure 6. The first, bounded, porous level considers the porous unit cell as if there are no inclusions present and contains one subdomain $\Omega_{e}^{(1)}$. The two unbounded levels consider the scattering of one of the inclusions in an unbounded porous domain. For the square scatterer of the first unbounded level, the unbounded problem is partitioned into a bounded and an unbounded region by truncation circle $\Gamma_{t, I_{e e}}^{(1,1)}$ with radius $R_{t_{e}}^{(1,1)}$. Within this truncation circle four bounded subdomains $\Omega_{e}^{(1,1, \gamma)}$ are constructed. As the second scatterer is a circle, the truncation circle $\Gamma_{t, v_{e}}^{(1,2)}$ coincides with the physical boundary and no additional bounded domains within the unbounded level are needed.

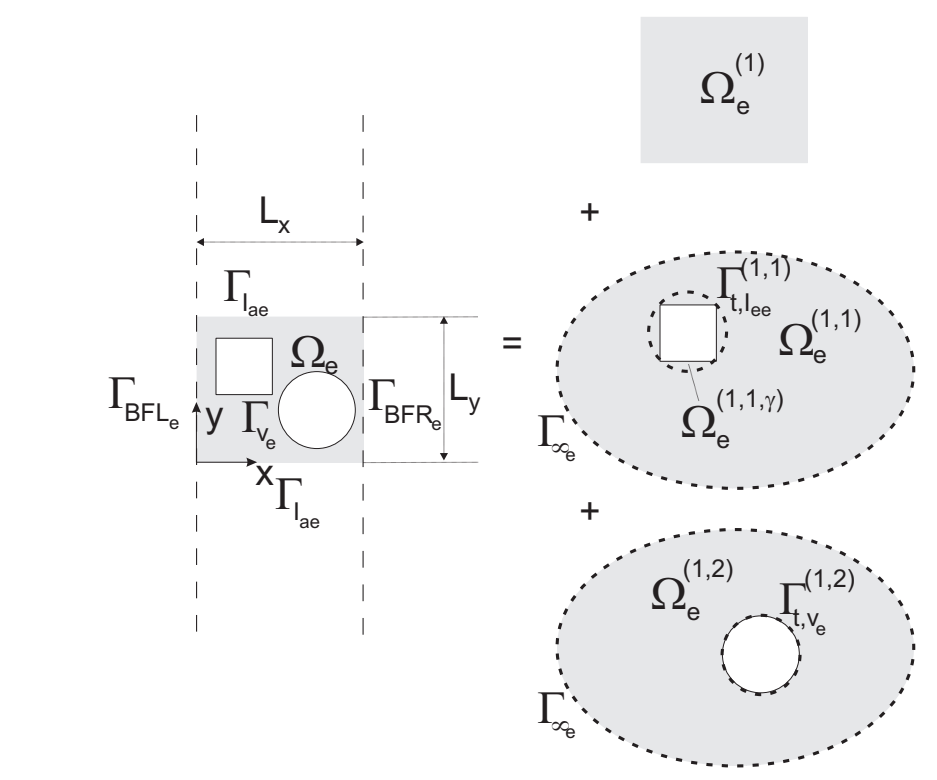

Figure 6: Multi-Level decomposition of the porous domain of the unit cell of Figure 5.
+ weighted residual

formulation

The pressure fields in the semi-unbounded acoustic domains are approximated using the novel wave function expansions, detailed in Section 4.2. The pressure fields of the porous subdomains within the different levels are approximated with bounded or unbounded wave functions, as explained in Section 3.2. The pressure field in the compound subdomain $\Omega_{e}^{\left(1^{\prime}\right)}$, is then constructed using the the Multi-Level expansion (10), while the pressure field in the bounded subdomains of the unbounded levels, $\Omega_{e}^{(\alpha, \beta, \gamma)}$ is approximated with regular wave functions for bounded problems. The compound subdomain $\Omega_{e}^{\left(1^{\prime}\right)}=\Omega_{e}^{(1)} \cap \Omega_{e}^{(1,1)} \cap \Omega_{e}^{(1,2)}$, of the unit cell problem of Figure 5 is shown in Figure 7. The pressure field within this domain is approximated using the bounded wave functions of the bounded level $\Omega_{e}^{(1)}$ augmented with the unbounded wave functions of the unbounded domains, $\Omega_{e}^{(1,1)}$ and $\Omega_{e}^{(1,2)}$ originating from the two unbounded levels, equation (10).

All boundary and interface conditions have to be minimised in an integral sense to obtain the system of equations. 


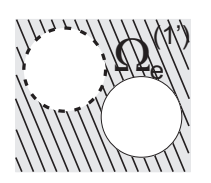

Figure 7: Compound domain $\Omega_{e}^{\left(1^{\prime}\right)}$ of the unit cell of Figure 5 .

All residuals on the boundaries of the acoustic subdomains $\Omega_{a}^{(\alpha)}$, the compound porous domain $\Omega_{e}^{\left(1^{\prime}\right)}$ and the bounded subdomains within the unbounded levels $\Omega_{e}^{(\alpha, \beta, \gamma)}$ are minimised in the following weighted residual formulation:

$$
\begin{aligned}
& -\int_{\Gamma_{B F L_{e}}^{\left(1^{\prime}\right)}} \mathcal{L}_{v_{e}}\left(\tilde{p}_{e}^{(1)}(\mathbf{r})\right) R_{B F L_{e}}\left(p_{e}^{\left(1^{\prime}\right)}(\mathbf{r})\right) \mathrm{d} \Gamma+\int_{\Gamma_{B F R_{e}}^{\left(1^{\prime}\right)}} \tilde{p}_{e}^{(1)}(\mathbf{r}) R_{B F R_{e}}\left(p_{e}^{\left(1^{\prime}\right)}(\mathbf{r})\right) \mathrm{d} \Gamma \\
& +\sum_{\alpha=1}^{2}\left(-\int_{\Gamma_{I_{a e}}^{\left(\alpha, 1^{\prime}\right)}} \mathcal{L}_{v_{e}}\left(\tilde{p}_{e}^{(1)}\right) R_{I p_{a e}}\left(p_{a}^{(\alpha)}(\mathbf{r}), p_{e}^{\left(1^{\prime}\right)}(\mathbf{r})\right) \mathrm{d} \Gamma+\int_{\Gamma_{I_{a e}}^{\left(\alpha, 1^{\prime}\right)}} \tilde{p}_{a}^{(\alpha)} R_{I v_{a e}}\left(p_{a}^{(\alpha)}(\mathbf{r}), p_{e}^{\left(1^{\prime}\right)}(\mathbf{r})\right) \mathrm{d} \Gamma\right) \\
& +\sum_{\beta=1}^{N_{u b}^{(1)}}\left(\int_{\Gamma_{t, v_{e}^{\left(1^{\prime}\right)}}} \tilde{p}_{e}^{(1, \beta)}(\mathbf{r}) R_{v_{e}}\left(p_{e}^{(1)}(\mathbf{r})\right) \mathrm{d} \Gamma+\sum_{\gamma=1}^{N_{b}^{(1, \beta)}}\left(\int_{\Gamma_{t, l_{e e}}^{\left(1^{\prime},(1, \beta, \gamma)\right)}} \tilde{p}_{e}^{(1, \beta)}(\mathbf{r}) R_{I Z_{e e}}\left(p_{e}^{\left(1^{\prime}\right)}(\mathbf{r}), p_{e}^{(1, \beta, \gamma)}(\mathbf{r})\right) \mathrm{d} \Gamma\right.\right.
\end{aligned}
$$

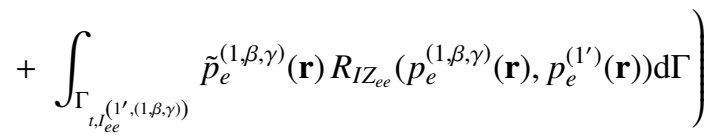

$$
\begin{aligned}
& \left.+\sum_{\gamma=1}^{N_{b}^{(1, \beta)}}\left(\int_{\Gamma_{t, v_{e}(1, \beta, \gamma)}} \tilde{p}_{e}^{(1, \beta, \gamma)}(\mathbf{r}) R_{v_{e}}\left(p_{e}^{(1, \beta, \gamma)}(\mathbf{r})\right) \mathrm{d} \Gamma+\sum_{\chi=1, \chi \neq \gamma}^{N_{b}^{(1, \beta)}} \int_{\Gamma_{t, l_{e e}^{((1, \beta, \gamma),(1, \beta, \gamma))}}} \tilde{p}_{e}^{(1, \beta, \gamma)}(\mathbf{r}) R_{I Z_{e e}}\left(p_{e}^{(1, \beta, \gamma)}(\mathbf{r}), p_{e}^{(1, \beta, \chi)}(\mathbf{r})\right) \mathrm{d} \Gamma\right)\right)=0 .
\end{aligned}
$$

The subscript associated to the boundaries along which the integral is taken is added to indicate on which subdomain the residual is considered. Each residual is written as a function of the pressure expansion(s) considered. The residuals (11)-(12) on the periodic boundaries (first line of equation (16)) are expressed using the compound pressure field of domain $\Omega^{\left(1^{\prime}\right)}$. The wave functions of the bounded level are used as weighting functions. When inclusions are present, this thus results in a coupling between the wave functions of the bounded level and the unbounded wave functions from the unbounded levels.

In a similar fashion, the coupling conditions between the two semi-unbounded acoustic and the porous domains (6) are accounted for (second line of equation (16)). The residuals are expressed using the compound pressure field expansion (10) for the field variables on the porous side and the pressure field expansion (7) for the semi-unbounded acoustic domain using the novel periodic, semi-unbounded wave functions. On the porous side, these residuals are weighted with the bounded wave functions of the bounded level resulting again in coupling matrices with the unbounded wave functions of the unbounded levels, but also with the acoustic wave functions. On the acoustic side, the weighting functions are expressed in terms of the semi-unbounded acoustic wave functions.

On the interfaces $\Gamma_{t, I_{e e}}^{\left(1^{\prime},(1, \beta, \gamma)\right)}$, continuity conditions between the compound subdomain $\Omega_{e}^{\left(1^{\prime}\right)}$ and the bounded subdomains of the unbounded levels $\Omega_{e}^{(1, \beta, \gamma)}$ are imposed (third line, second term and fourth line of (16)). On the side of the compound subdomain, these are weighted with the unbounded wave functions of the level $\beta$, belonging to that 
inclusion; on the side of the bounded domain of the unbounded level, $\Omega^{(1, \beta, \gamma)}$, these are weighted with these bounded wave functions.

The residuals on the rigid part of the truncation surfaces $\Gamma_{t, v_{e}}^{(1, \beta)}$, facing the compound subdomain $\Omega_{e}^{\left(1^{\prime}\right)}$ are expressed using the compound pressure expansion and weighted with the unbounded wave functions of $\Omega^{(1, \beta)}$ (third line of (16), first term). This is e.g. needed for the rigid boundary conditions on the circular inclusion. When the inclusion is non-circular, a part of its boundary is also adjacent to bounded domains of the unbounded levels. The residual as well as the weighting functions are expressed using the bounded wave functions of that unbounded level (first term of the last line of (16)). For coupling conditions on the interface $\Gamma_{I_{e e}}^{((1, \beta, \gamma),(1, \beta, \chi))}$ between two bounded subdomains $\Omega^{(1, \beta, \gamma)}$ and $\Omega^{(1, \beta, \chi)}$ within an unbounded level (not needed in Figure 6), the bounded wave functions of the unbounded level are used to approximate the interface residuals and as weighting functions (last line of (16), second term)).

The structure of the resulting system of equations in given and discussed in Appendix D

All WBM routines are implemented in Matlab R2013b. The system of equations is solved using Gaussian elimination.

\subsection{Reflection, transmission and absorption coefficient evaluation}

When the system of equations of the unit cell model is solved for the contribution factors of the wave functions, dynamic fields of interest can be post-processed. Due to the plane wave nature of the wave functions in the semiunbounded periodic acoustic domains, the hemispherical reflection and transmission coefficients, $\mathcal{R}$ and $\mathcal{T}$, can be straightforwardly calculated based on the wave function contribution factors $p_{a, w}^{(\bullet)}$ of the semi-unbounded acoustic wave functions and the selected wave number components, Equation (15):

$$
\begin{aligned}
\mathcal{R} & =\sum_{w} \frac{\mathfrak{R}\left(k_{B F_{y w}}^{(1)}\right)\left\|p_{a, w}^{(1)}\right\|^{2}}{k_{a y}\|A\|^{2}}, \\
\mathcal{T} & =\sum_{w} \frac{\mathfrak{R}\left(k_{B F_{y w}}^{(2)}\right)\left\|p_{a, w}^{(2)}\right\|^{2}}{k_{a y}\|A\|^{2}},
\end{aligned}
$$

accounting for the power of all propagating waves in the semi-unbounded wave function expansion. $A$ is the amplitude of the incoming plane wave, see Appendix C.3.

The absorption coefficient $\mathcal{A}$ cannot be straightforwardly evaluated using wave function contribution factors. It can, however, be evaluated using the conservation of energy:

$$
\mathcal{A}=1-\mathcal{R}-\mathcal{T}
$$

\section{Numerical verification examples}

This section shows the potential of the novel WBM for unit cell models for four problem cases. The first example considers a problem with an exact analytical solution available: a plane wave impinging on a rigidly backed infinite porous layer. This way the correctness and efficiency of the WBM implementation is validated. 
The next example considers an absorption case that has been presented in literature: the obtained results are compared to existing MPM calculations. The third validation example studies the same problem and an extension with two circular inclusions in a sound transmission context. Again, the method is validated with MPM calculations. The final verification case considers a C-shape inclusion, which can be efficiently studied using the WBM, contrary to the MPM. As previously mentioned, the meshless nature of the WBM allows for an easy optimisation procedure for the problem at hand. This is also illustrated for this final example.

\begin{tabular}{ll}
\hline \multicolumn{2}{c}{ Air properties } \\
\hline Fluid density & $\rho_{a}=1.213 \mathrm{~kg} / \mathrm{m}^{3}$ \\
Ambient Pressure & $P_{0}=1.01325 \cdot 10^{5} \mathrm{~Pa}$ \\
Ratio of specific heats & $\gamma=1.4$ \\
Fluid dynamic viscosity & $\eta=1.839 \cdot 10^{-5} \mathrm{~kg} /(\mathrm{ms})$ \\
\hline \multicolumn{1}{c}{ Fireflex material properties } \\
\hline Porosity & $\phi=0.95$ \\
Viscous characteristic length & $\Lambda=1.8 \cdot 10^{-4} \mathrm{~m}$ \\
Thermal characteristic length & $\Lambda^{\prime}=3.6 \cdot 10^{-4} \mathrm{~m}$ \\
Static flow resistivity & $\sigma=8.9 \cdot 10^{3} \mathrm{~kg} /\left(\mathrm{m}^{3} \mathrm{~s}\right)$ \\
Tortuosity & $\alpha_{\infty}=1.42$
\end{tabular}

Table 1: Material properties of air and Fireflex [8].

A Fireflex foam material is considered in the verification cases. The material data of this foam and the saturating air are obtained from literature [8], and are listed in Table 1.

\subsection{Verification case: 'Invisible' inclusion}
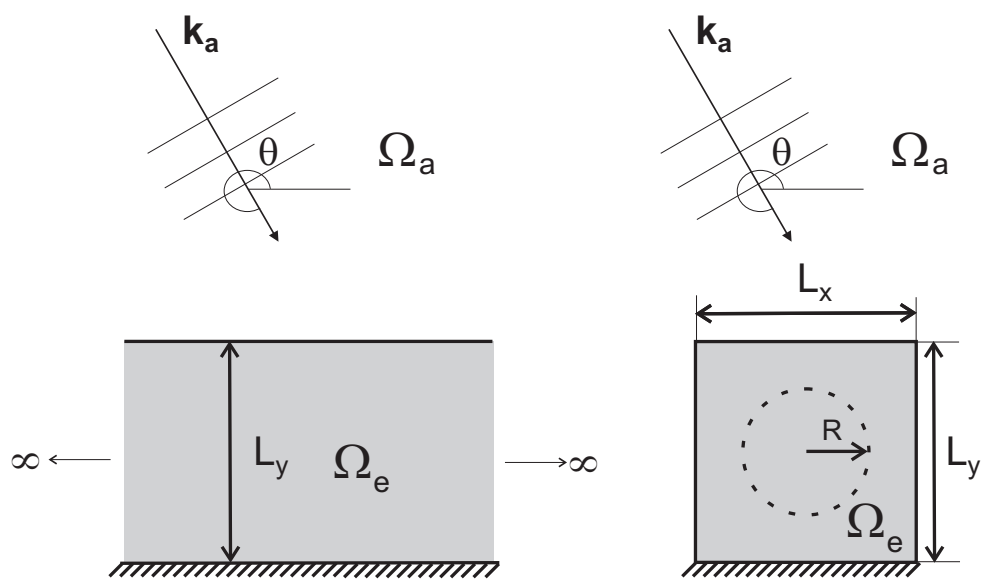

Figure 8: Left: plane wave impinging under angle of incidence $\theta$ on a porous rigidly backed layer (left), right: WB unit cell model with an 'invisible' circular inclusion.

The first verification case considers a plane wave impinging on an infinitely long porous material of thickness $L_{y}$, backed by a rigid plate, see Figure 8, left. This problem can be solved analytically [16] and thus can be used to evaluate the implementation and the convergence of the unit cell WBM with inclusions. The same wave field should be found 
by considering a WB unit cell model with the same geometry, material properties and angle of incidence, as indicated on the right hand side of Figure 8. The length of the unit cell $L_{x}$ can be chosen arbitrarily in this case. To evaluate the ML implementation, an 'invisible' inclusion is considered: the circular inclusion with radius $R$ is composed of exactly the same material as the surrounding porous material. On the resulting truncation circle, subdomain coupling conditions (B.1) are imposed between the wave functions of the resulting bounded domain of the unbounded level and the compound wave function set exterior to the truncation circle. A square unit cell of $2 \mathrm{~cm}$ by $2 \mathrm{~cm}$ is considered. The 'invisible' inclusion has a radius of $3.5 \mathrm{~mm}$ and is centered in the middle of the unit cell.

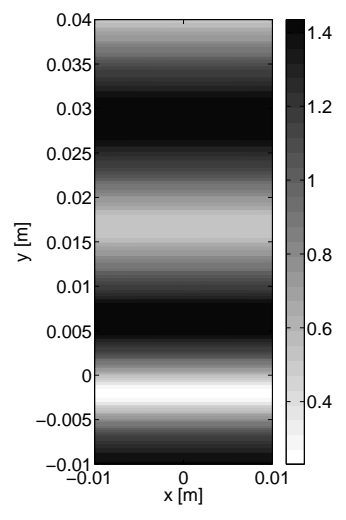

(a) Pressure amplitude $[\mathrm{Pa}]$ by the WBM.

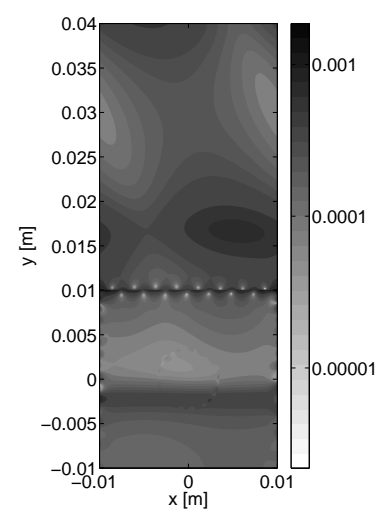

(b) Relative error $\epsilon[-]$ of the WBM (ref. analytical solution).

Figure 9: Pressure amplitude [Pa] (a) and relative error (b) at $10 \mathrm{kHz}$, angle of incidence $\pi / 4$.

Figure 9(a) shows the absolute value of the pressure fields at $10 \mathrm{kHz}$ when the considered angle of incidence is $-\pi / 4$ calculated by the WBM and evaluated in the porous layer (from $-0.01 \mathrm{~m}$ to $0.01 \mathrm{~m}$ ) as well as in the acoustic domain above the foam. The pressure fields are clearly continuous. The WBM model uses a truncation factor $N=10$ : all wave functions with wave numbers smaller or equal to 10 times the largest physical wave number are included, leading to a total of 128 wave functions (15 in the semi-unbounded acoustic domain, 64 in the bounded level, 21 in the unbounded domain and 28 in the bounded domain of the unbounded level). Figure 9(b) shows the logarithm of the absolute value of the relative error of the same pressure field with respect to the analytical solution, calculated as:

$$
\varepsilon\left(p_{\bullet}(\mathbf{r})\right)=\frac{\left|p_{\bullet}(\mathbf{r})-p_{\bullet}^{r e f}(\mathbf{r})\right|}{\left|p_{\bullet}^{r e f}(\mathbf{r})\right|}
$$

Accurate results are obtained, with relative errors less than 1\%. Larger errors are mainly present at the boundaries, where residuals are minimised.

The top of Figure 10 shows the analytically obtained absorption coefficient when the angle of incidence is $-\pi / 9$ and the absorption curve calculated by the WBM for the problem setting with the invisible circle as described above. For the WBM calculations, a truncation factor $N=30$ is applied, resulting in a total of 22 wave functions at $100 \mathrm{~Hz}$ and 736 wave functions at $22 \mathrm{kHz}$. Both curves are on top of each other. The bottom of Figure 10 shows the absolute 
value of the relative error between the WBM prediction and the analytical solution:

$$
|\varepsilon|=\frac{\left|\mathcal{A}-\mathcal{A}^{r e f}\right|}{\left|\mathcal{A}^{r e f}\right|} .
$$

Over the complete frequency band of interest, running from $100 \mathrm{~Hz}$ to $22 \mathrm{kHz}$, excellent accuracies are obtained. Using the given settings, the calculations take less than $1 \mathrm{~ms}$ per frequency line, run on a $2.10 \mathrm{GHz}$ Intel Dual Core i7 processor and 16GB ram, with a Windows 7 operation system.
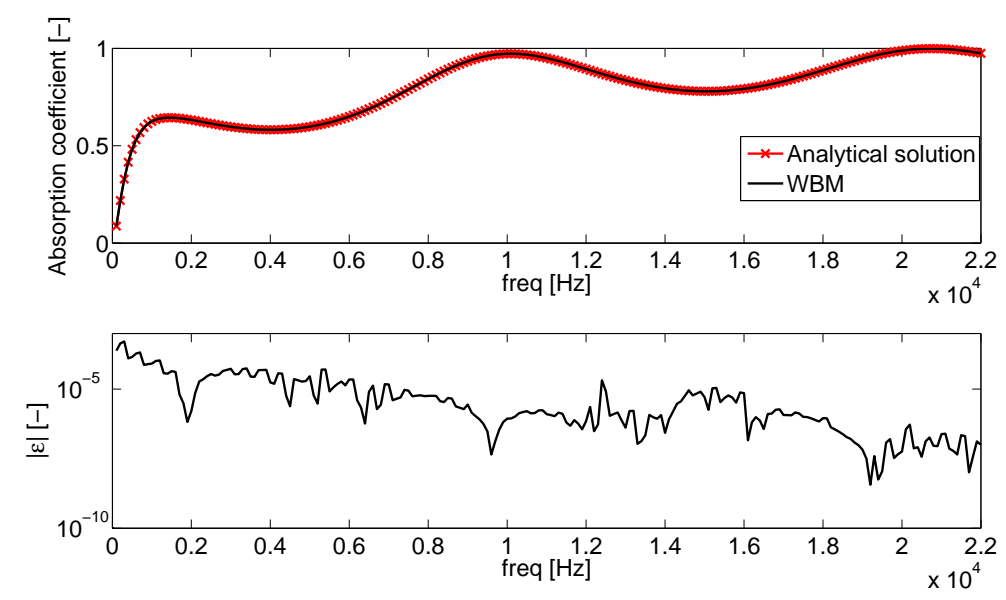

Figure 10: Top: Absorption coefficient, angle of incidence $-\pi / 9$ calculated by WBM and the analytical solution. Bottom: relative error between both calculations.

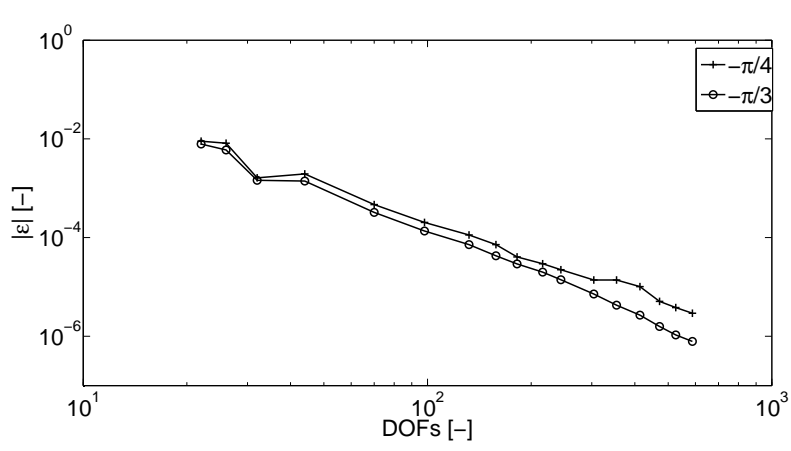

(a) $5 \mathrm{kHz}$

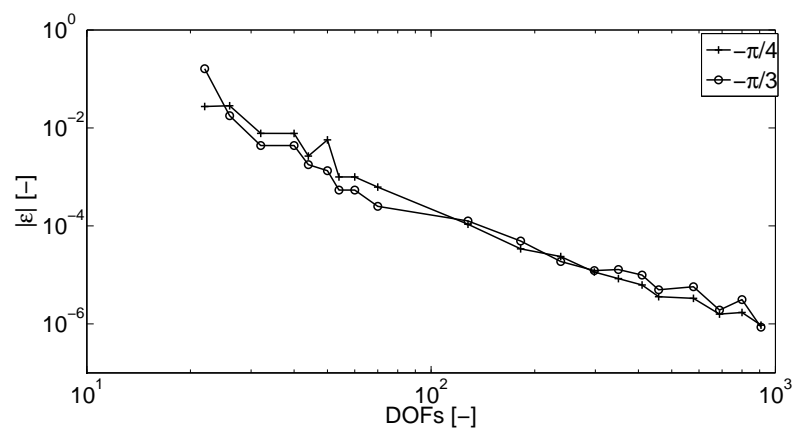

(b) $10 \mathrm{kHz}$

Figure 11: Convergence studies of the WBM with an invisible circle, considering angles of incidence of $\pi / 4(+)$ and $\pi / 3(\circ)$ at two different frequencies.

Finally, a convergence study is performed for different angles of incidence $(-\pi / 3$ and $-\pi / 4)$ and different frequencies of interest $(5 \mathrm{kHz}$ and $10 \mathrm{kHz}$ ). The absorption coefficient is calculated using increasingly refined WB models: the number of wave functions are gradually increased from 22 to 588 at $5 \mathrm{kHz}$ and from 22 to 908 at $10 \mathrm{kHz}$, applying truncation factors of $N=0.4$ to $N=80$ at $5 \mathrm{kHz}$ and to $N=60$ at $10 \mathrm{kHz}$. Figure 11 shows the obtained relative accuracies as a function of the number of degrees of freedom (DOFs). High convergence rates are obtained, irrespective of 
the angle of incidence and the considered frequency.

\subsection{Validation case: Absorption}

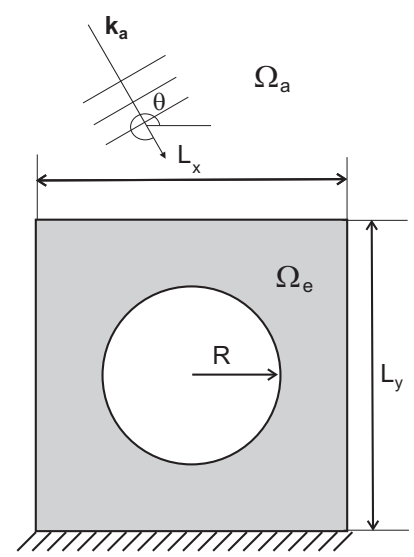

Figure 12: Acoustic-porous unit cell problem geometry with a circular rigid inclusion and a rigid backing.

The next validation case applies the WBM to obtain the absorption coefficient of a simple, rigidly-backed porous unit cell with a single circular inclusion as shown in Figure 12. The dimensions, the frequency range as well as the material properties are based on values available from literature [8]. The thickness $L_{y}$ of the porous medium is equal to the distance between the inclusions $L_{x}$ and is taken $2 \mathrm{~cm}$. The inclusions are rigid, are centered in the unit cell, and have a radius $R$ of $7.5 \mathrm{~mm}$. A plane wave, incident under angle $\theta$, impinges on the structure.

The acoustic absorption coefficient is calculated for the frequency range of $100 \mathrm{~Hz}$ to $18 \mathrm{kHz}$ and for four different angles of incidence using the WBM and the MPM. Figure 13 shows the result of the different simulations. The WBM and MPM curves are on top of each other, indicating the correctness of the WB results. As discussed in [8], the

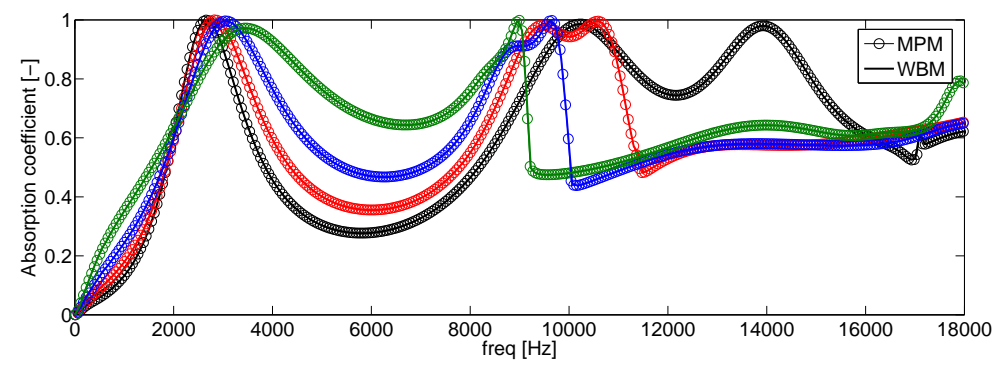

Figure 13: Absorption coefficient for the foam with periodic circular inclusions under four different angles of incidence: black: $-\pi / 2$, red: $-\pi / 3$, blue: $-\pi / 4$, green: $-\pi / 6$.

addition of periodically distributed, high contrast inclusions leads to a high absorption coefficient in the low-frequency range, below the expected $\lambda / 4$ absorption peak, which would occur around $4 \mathrm{kHz}$ for normal incidence. Due to the rigid inclusion and the rigid backing, entrapment of energy occurs at a lower frequency of around $2700 \mathrm{~Hz}$. Figure 14 visualises the absolute value of the pressure field at this frequency, considering normal incidence. The figure clearly 
shows the trapped mode, calculated by the WBM, and in agreement with literature [8]. Besides, high absorption coefficients are obtained at higher frequencies, which correspond to trapped modes or modified modes of the porous material, excited thanks to periodic inclusions. The absorption coefficient has a minimum at the first Bragg frequency around $6 \mathrm{kHz}$. The low frequency behaviour of the considered unit cell forms yet a starting point; the following examples discuss the transmission loss of similar structures, focussing on low-frequency transmission and normal incidence.

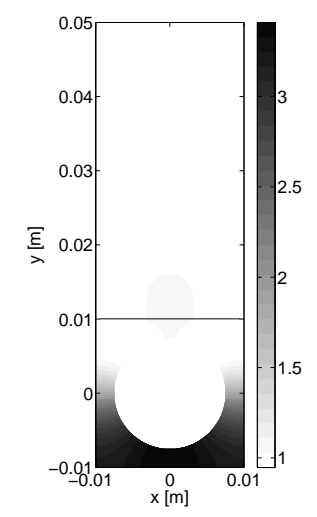

Figure 14: Absolute value of the pressure field in the air and porous domain at a frequency of $2700 \mathrm{~Hz}$ and considering an angle of incidence of $-\pi / 2$.

\subsection{Validation case: Transmission - multiple inclusions}

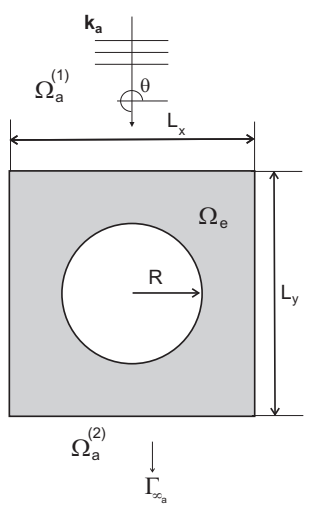

(a) Unit cell with a circular rigid inclusion.

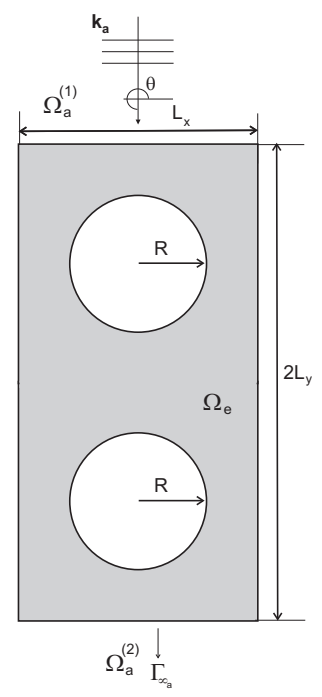

(b) Double-thickness unit cell with two circular rigid inclusions.

Figure 15: Acoustic-porous-acoustic unit cell problem geometries for transmission calculations. 
The next validation case considers two unit cell geometries, shown in Figure 15. Figure 15(a) considers the same problem setting as the previous validation case, however, in a transmission context. Dimensions are unchanged and the angle of incidence is $-\pi / 2$. As the interaction between the rigid inclusion and the rigid backing is no longer present, no peak in absorption around $2700 \mathrm{~Hz}$ is expected. For this reason, and also to validate the WBM implementation for multiple inclusions, the geometry of Figure 15(b) is studied. The thickness of the porous layer is doubled and contains two rigid inclusions at a distance $L_{y}$ of each other, centered according to the $x$-direction. This configuration allows for entrapment of energy between both inclusions, in a similar way as in the previous validation case.
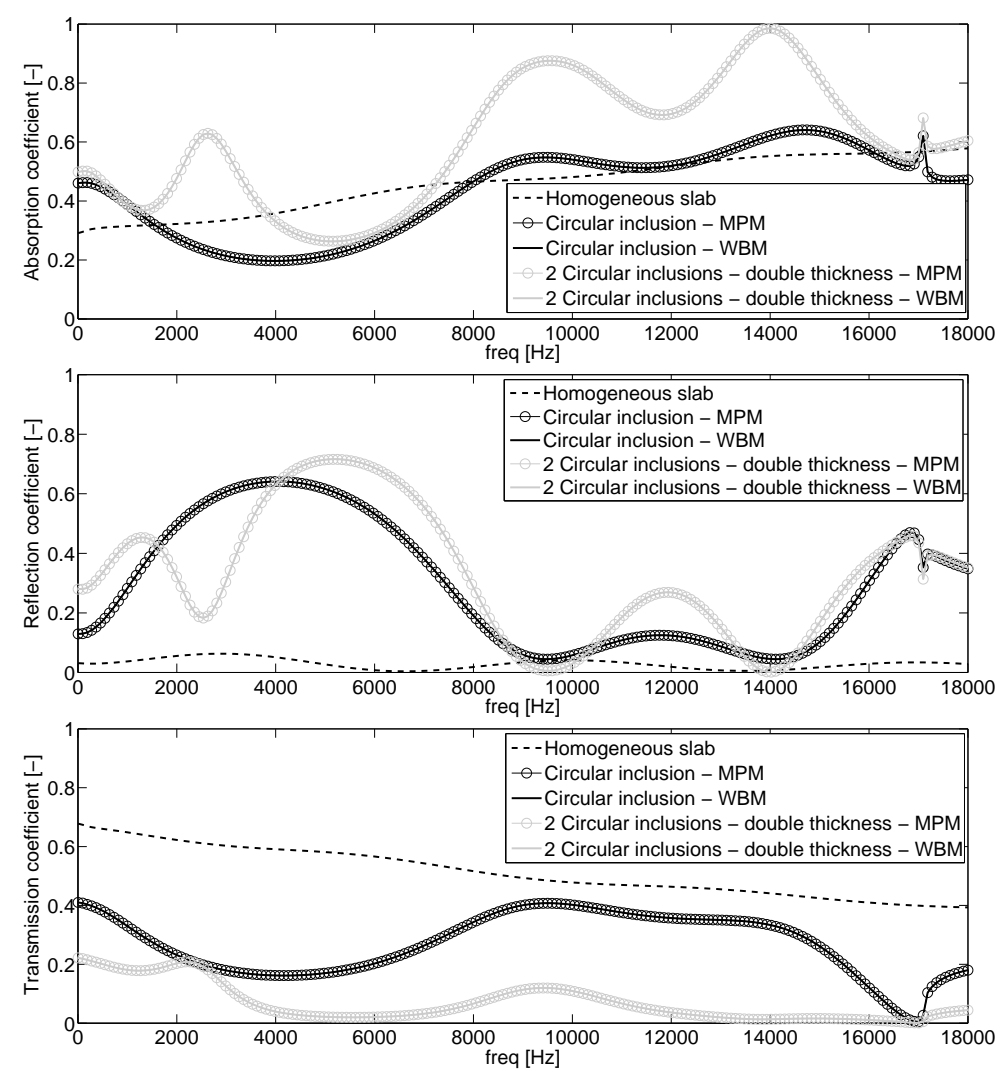

Figure 16: Absorption coefficient (top), reflection coefficient (middle) and transmission coefficient (bottom) for the Fireflex foam with and without circular inclusions.

Figure 16 shows the absorption, reflection, and transmission coefficient of the periodic medium, with and without periodic rigid circular inclusions, calculated with the WBM and the MPM for audible frequencies between $5 \mathrm{~Hz}$ and $18 \mathrm{kHz}$. Again, a perfect match between the WBM and the MPM is seen, confirming the validity of the former. When comparing the results of the single periodic inclusion (black solid lines) to the ones for the homogeneous porous material (black dashed line), no major changes are seen in the absorption coefficient at low frequencies. The reflection coefficient, however, is clearly increased due to the presence of the rigid inclusions. This is reflected in a decreased transmission coefficient. At higher frequencies, there are regions of improved absorption, due to the modified modes 


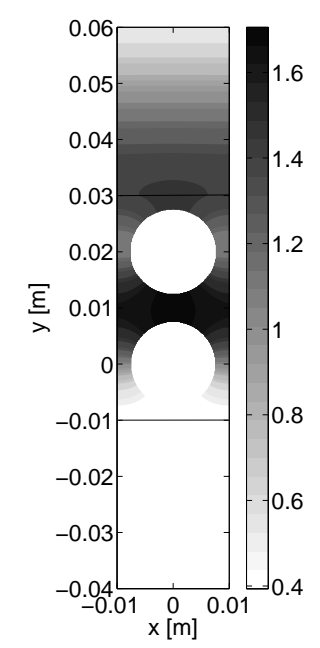

Figure 17: Absolute value of the pressure field in the air and porous domains for the unit cell with two inclusions at a frequency of $2700 \mathrm{~Hz}$ and considering an angle of incidence of $-\pi / 2$.

of the porous material that are excited by the grating [8]. When the second unit cell, with two inclusions and double thickness, is considered, an improved absorption coefficient, and consequently an improved transmission coefficient, is obtained. Due to the thicker material, of course, the absorption coefficient increases, but also similar effects as in the previous validation case are seen: a high peak in the absorption coefficient around $2700 \mathrm{~Hz}$ due to entrapment of energy between both inclusions.

To confirm this statement, Figure 17 shows a contour plot of the absolute value of the pressure in the acoustic and porous domains calculated by the WBM at $2700 \mathrm{~Hz}$. A clear maximum is seen between both inclusions and the pressure field around both inclusions are very similar to the one obtained in the previous validation case. The pressure fields between the air and the porous layer are also continuous.

\subsection{Application case: $C$-shaped inclusion}

Although the unit cell with two circular inclusions provides an improved transmission coefficient, the thickness of the layer has been doubled, being a more bulky solution. The final application case considers a slightly more complex inclusion shape, namely a thin C-shape. The problem setting is illustrated in Figure 18 applying the same material properties, dimensions, excitation and frequency range as in the previous validation case with a single inclusion per unit cell. The thickness $e$ of the $\mathrm{C}$-shape is $0.5 \mathrm{~mm}$, the outer radius is $7.5 \mathrm{~mm}$ and the inner radius is $7 \mathrm{~mm}$. The aperture of the $\mathrm{C}$-shape is indicated by the angle $\varphi$. The $\mathrm{C}$-shape is assumed to be rigid, i.e. Neumann boundary conditions are applied. For non-circular inclusions, the MPM cannot be straightforwardly applied. The WBM on the other hand, is still applicable. The unit cell is subdivided in one bounded and one unbounded level. The bounded domain within the WB truncation circle is partitioned into two subdomains according to the C-shape, as indicated in Figure 19. The associated subdomain bounding boxes are indicated in thin lines, following the inner geometry. 


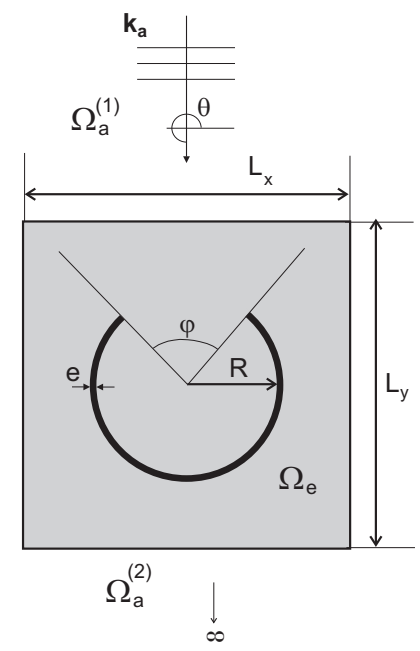

Figure 18: Acoustic-porous-acoustic unit cell problem geometry with a C-shaped rigid inclusion.

Figure 19: Partitioning of the bounded subdomains inside the trunction surface and bounding boxes.

To illustrate the effect of the C-shape inclusion, an angle of aperture $\varphi=\pi / 2$ is chosen. Figure 20 shows the obtained absorption, reflection and transmission coeffificient as compared to the results of the previous transmission case calculations. As compared to the single çircular.inclusion, an in general higher absorption coefficient is obtained, as more porous material is available in the (unit cẹt,,$; 1 ;)$ the peaks in the absorption coefficient, due to modified porous modes, excited by the periodic grating, are still present A large, additional peak is seen around $4500 \mathrm{~Hz}$. This one is the result of a local mode of the inclusion. This is validated in Figure 21, showing the absolute value of the pressure field at this frequency, in the air as well as in the porous material, calculated by the WBM. Due to the higher absorption coefficient an improved transmission coefficient is obtained as compared to the single circular inclusion problem.

As a final demonstration, an optimisation case is carried out to show the potential of the proposed method in such scenarios. Various design scenarios can be followed in order to optimise the transmission/absorption characteristics of the periodic inclusions. For instance, the optimal position and orientation of the inclusion can be investigated. Moreover, the shape of the inclusion can be optimised. With the presented method, it is possible to optimise the aperture angle of the $\mathrm{C}$-shape to tune local resonances (as in [41] for acoustic lenses). The execution of such design scenarios is conceptually easy within the WBM as the domain does not need to be remeshed for each iteration and the calculations are computationally not demanding. Furthermore, the system matrix of the unit cell can be reused for different iteration steps; only the coupling matrices and (in the case of shape optimisation) the system matrix of 

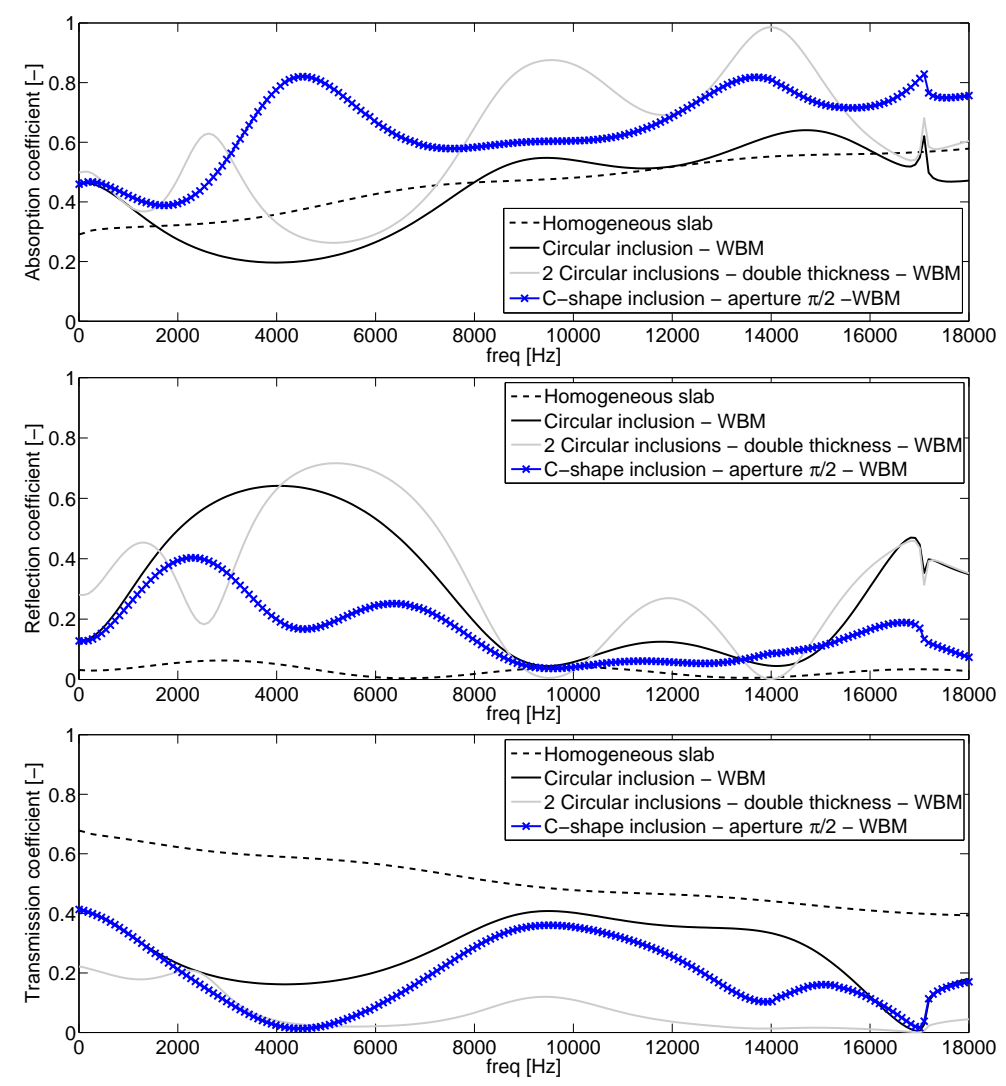

Figure 20: Absorption coefficient (top), reflection coefficient (middle) and transmission coefficient (bottom) for the Fireflex foam with and without circular inclusions and C-shape inclusions with an aperture of $\pi / 2$.

the inclusion have to be recalculated. Because of those advantages, the unit cell modelling with the WBM becomes a very appealing tool for the optimisation of periodic inclusions.

As an illustration, the aperture of the $\mathrm{C}$-shape, $\varphi$, is optimised for a low transmission coefficient at a single target frequency of $2700 \mathrm{~Hz}$, selected based on the previous validation cases. The goal is thus to obtain a minimised transmission coefficient, using an equally thin porous layer as considered for the absorption case, by optimising the angle of aperture $\varphi$.

A genetic algorithm (Matlab Optimisation toolbox) has been used to find the optimum angle to obtain a minimised transmission coefficient; in practice $1 / \mathcal{T}$ has been maximised. It should be noted that the current example mainly serves to indicate the potential of the periodic WBM for optimisation studies. Details on the convergence, the population size, number of iterations, etc. are omitted since it is out of scope for this paper. The optimisation procedure leads to optimal angle $\varphi$ of 0.4746 radians.

Figure 22 shows the obtained absorption, reflection and transmission coefficients when the retrieved optimal angle is used in the WBM unit cell model as compared to the previous calculations. It is seen that the dip in the transmission coefficient indeed shifts to lower frequencies and that this effect is mainly due to an (again) increased absorption 


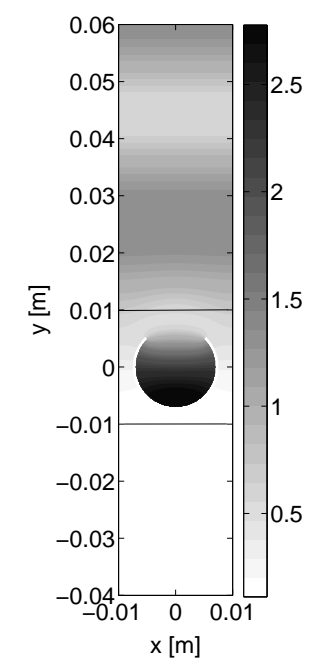

Figure 21: Absolute value of the pressure field in the air and porous domains at a frequency of $25 \mathrm{kHz}$ and considering an angle of incidence of $\pi / 2$ and an aperture angle $\varphi$ of $\pi / 2$ radians.

coefficient. Figure 23 shows the absolute value of the associated pressure field at that particular frequency.

Although further optimisations can be performed, this example serves as a good first validation of the potential of the developed WBM unit cell approach.

\section{Conclusions and outlook}

This paper presents an extension to the Wave Based Method to allow for efficient absorption, reflection and transmission coefficient calculations of porous materials with periodic inclusions. The porous material is modelled as an equivalent fluid. The Multi-Level Wave Based Method is extended to account for periodicity conditions, modelling a unit cell of the periodic structure. A novel periodic semi-unbounded wave function set is presented for the acoustic domains, which satisfies the periodicity conditions, the Helmholtz equation and the Sommerfeld radiation condition inherently. The method is first validated for a problem which has an exact analytical solution and a high convergence rate is shown. The method is also applied to an absorption problem with rigid circular inclusions, available in literature. By comparing the WBM results to MPM results its validity and accuracy are shown. Based on these results, also transmission cases have been defined, considering one and two inclusions per unit cell. The method is, however, not restricted to circular inclusion geometries. As a final example, C-shaped inclusions are studied. The Wave Based Method is meshless and computationally efficient, which makes it ideal for optimisation studies. The aperture of a $\mathrm{C}$-shape inclusion is optimised for a low transmission coefficient at a dedicated frequency.

Future steps include the extension of the presented method towards three-dimensional problems. Similar steps have to be taken as compared to the current paper: Bloch-Floquet conditions have to be imposed on all periodic boundaries of the unit cell, and three-dimensional semi-unbounded periodic acoustic wave functions need to be used. In addition, the methodology can be extended to consider poro-elastic unit cells with inclusions, of which the solid 

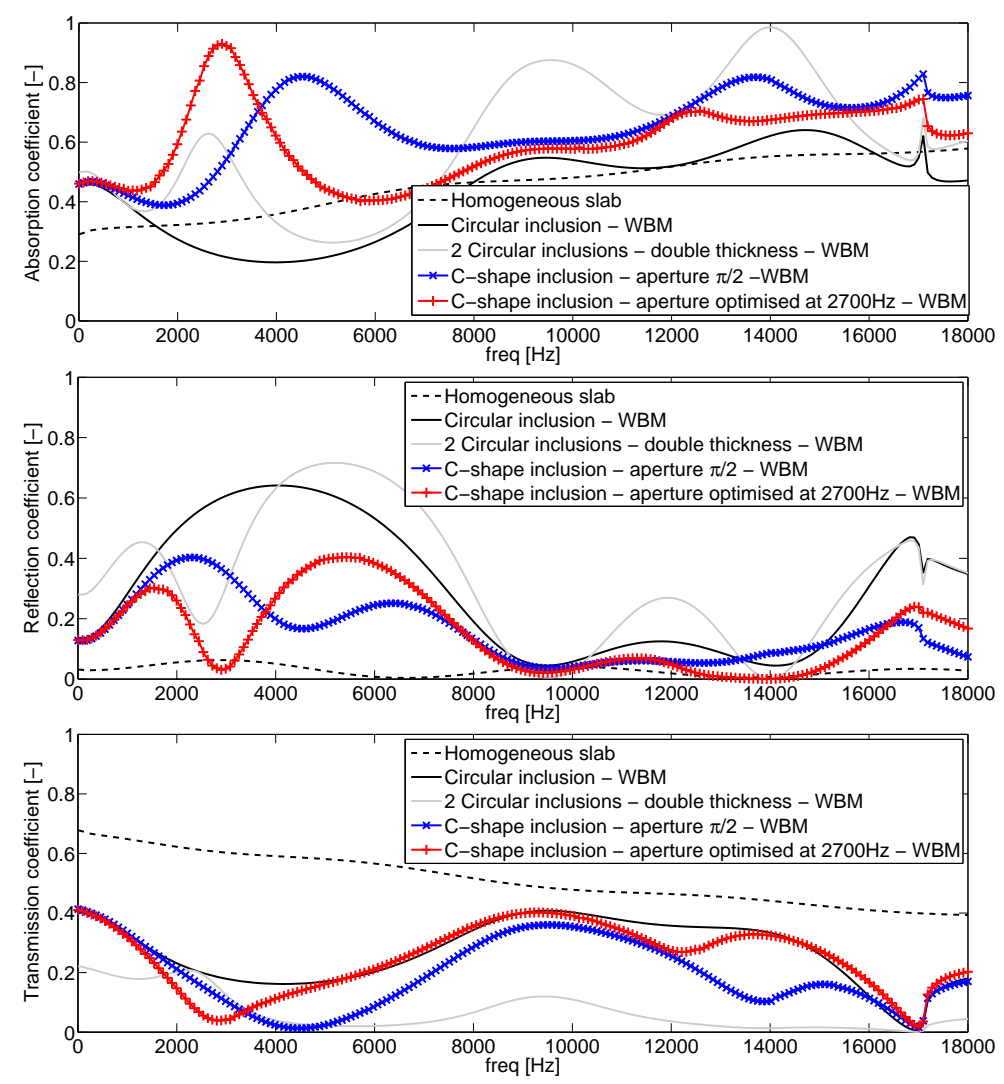

Figure 22: Absorption coefficient (top), reflection coefficient (middle) and transmission coefficient (bottom) for the Fireflex foam with and without circular inclusions.

skeleton is no longer considered rigid. The following strategy can be used. First, the Multi-Level concept needs to be evaluated for poro-elastic WB models: unbounded poroelastic wave functions need to be defined. In a next step, Bloch-Floquet boundary conditions, linking all solid and fluid field variables on periodic interfaces need to be included as well as the coupling with semi-unbounded periodic acoustic domains.

\section{Acknowledgements}

Elke Deckers is a Postdoctoral Fellow of the Fund for Scientific Research-Flanders (F.W.O.), Belgium. The Research Fund KU Leuven is gratefully acknowledged for its support and the European Commission is gratefully acknowledged for their support of the ENLIGHT research project. The COST Action TU1105 NVH analysis techniques for design and optimization of hybrid and electric vehicles is gratefully acknowledged to finance a Short Term Scientific Mission of the first author to Le Mans. 


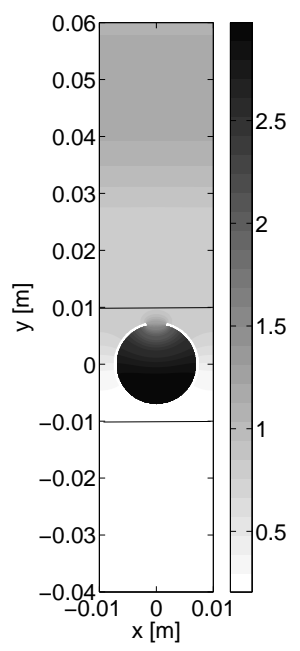

Figure 23: Absolute value of the pressure field in the air and porous domains at a frequency of $25 \mathrm{kHz}$ and considering an angle of incidence of $-\pi / 2$ and an aperture angle $\varphi$ of 0.4746 radians.

\section{Appendix A. Johnson-Champoux-Allard model of the equivalent fluid}

According to the Johnson-Champoux-Allard model the effective equivalent fluid density $\rho_{e}$ of the rigid frame equivalent fluid model can be written as:

$$
\rho_{e}(\omega)=\frac{\rho_{a} \alpha(\omega)}{\phi}
$$

where $\phi$ is the porosity of the porous material and $\alpha(\omega)$ is the dynamic tortuosity [14]. It accounts for frequencydependent viscous effects:

$$
\alpha(\omega)=\alpha_{\infty}\left[1+\frac{\sigma \phi}{\mathrm{j} \omega \rho_{a} \alpha_{\infty}} G_{J}(\omega)\right],
$$

where $\sigma$ is the flow resistivity, $\alpha_{\infty}$ the tortuosity and $G_{J}(\omega)$ is a relaxation function which takes into account the transition from microscale Stokes flow at very low frequencies to inviscid flow as high frequency asymptote:

$$
G_{J}(\omega)=,
$$

where $\Lambda$ is the viscous characteristic length [14].

In a similar way as for viscous effects, a function $\alpha^{\prime}(\omega)$ can be used to account for thermal effects in the effective equivalent bulk modulus $K_{e}$ of the rigid frame equivalent fluid model [42]:

$$
K_{e}(\omega)=\frac{\gamma P_{0}}{\phi\left(\gamma-(\gamma-1)\left(\alpha^{\prime}(\omega)\right)^{-1}\right)},
$$

where $\gamma$ is the specific heat ratio and $P_{0}$ is the ambient pressure. Champoux and Allard [15] have shown that the equivalent bulk modulus is controlled by different aspects of the pore geometry, introducing the thermal characteristic length $\Lambda^{\prime}$. Following their approach, $\alpha^{\prime}(\omega)$ is given by:

$$
\alpha^{\prime}(\omega)=1+\frac{8 \eta}{\mathrm{j} \Lambda^{\prime 2} \operatorname{Pr} \omega \rho_{a}},
$$

where $\operatorname{Pr}$ is the Prandtl number and $\eta$ is the fluid viscosity. 


\section{Appendix B. Subdomain coupling conditions}

On the interface $\Gamma_{I_{\bullet}}$ between two adjacent subdomains $\Omega_{\bullet}^{(\alpha)}$ and $\Omega_{\bullet}^{(\beta)}$, containing the same medium, continuity conditions need to be imposed. The continuity between pressure and displacement can be directly enforced, as in (6). Pluymers [37], however, has shown that applying impedance coupling conditions has a beneficial effect on the WBM's efficiency as artificial damping is added to the system and singularities which can occur at the eigenfrequencies of the associated subdomain are avoided. The residual $R_{I Z \text {.. }}^{(\alpha, \beta)}$ reads:

$$
\mathbf{r} \in \Gamma_{I_{\bullet \bullet}^{(\alpha, \beta)}}: R_{I Z \cdot \bullet}^{(\alpha, \beta)}=\left(\frac{\mathrm{j}}{\rho_{\bullet} \omega} \frac{\partial p_{\bullet}^{(\alpha)}(\mathbf{r})}{\partial n^{(\alpha)}}-\frac{p_{\bullet}^{(\alpha)}(\mathbf{r})}{\bar{Z}_{\bullet} \text { int }}\right)+\left(\frac{\mathrm{j}}{\rho_{\bullet} \omega} \frac{\partial p_{\bullet}^{(\beta)}(\mathbf{r})}{\partial n^{(\beta)}}+\frac{p_{\bullet}^{(\beta)}(\mathbf{r})}{\bar{Z}_{\bullet} \text { int }}\right)=0
$$

with $n^{(\alpha)}$ the local normal on the subdomain interface $\Gamma_{I_{. .}}$, outwards of domain $\Omega_{\bullet}^{(\alpha)}$ and $\bar{Z}_{\bullet}$ int an impedance coupling factor, typically chosen as $\rho_{\bullet} c_{\bullet}$, thus applying the characteristic impedance of the fluid. A similar coupling is applied in [43]. This continuity condition is enforced on each subdomain, for each interface, yielding two continuity conditions over each subdomain interface.

\section{Appendix C. Wave function selection}

This appendix gives the wave functions that are applied in two-dimensional bounded and unbounded subdomains and an expression for the particular solution term when a plane wave excitation is considered.

\section{Appendix C.1. Wave functions for a bounded subdomain}

For a $2 \mathrm{D}$ bounded subdomain $\Omega_{\bullet}^{(\alpha)}$, two types of wave functions are distinguished, the so-called r- and s-set:

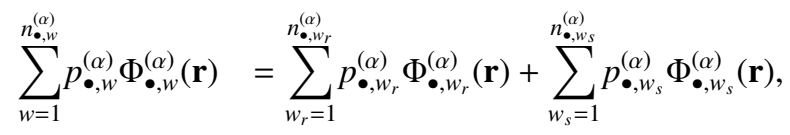

with $n_{\bullet, w}^{(\alpha)}=n_{\bullet, w_{r}}^{(\alpha)}+n_{\bullet, w_{s}}^{(\alpha)}$. These wave functions are defined as:

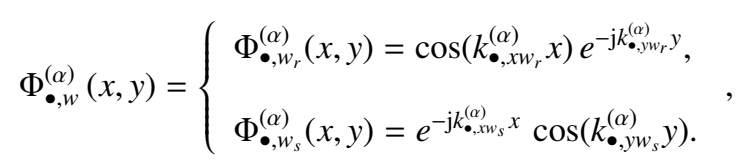

using a local coordinate system attached to the considered subdomain as shown in Figure C.24.

These wave functions (C.2) are selected to be exact solutions of the homogeneous part of the Helmholtz equation (1). Desmet [18] has shown that the following selection of wave number components lead to a converging wave function set:

$$
\begin{aligned}
& \left(k_{\bullet, x w_{r}}^{(\alpha)}, k_{\bullet, y w_{r}}^{(\alpha)}\right)=\left(\frac{w_{1_{\bullet}}^{(\alpha)} \pi}{L_{x_{\bullet}}^{(\alpha)}}, \pm\right), \\
& \left(k_{\bullet, x w_{s}}^{(\alpha)}, k_{\bullet, y w_{s}}^{(\alpha)}\right)=\left( \pm, \frac{w_{2_{\bullet}}^{(\alpha)} \pi}{L_{y_{\bullet}}^{(\alpha)}}\right) .
\end{aligned}
$$


Figure C.24: Illustration of the coordinate system and bounding box of a bounded subdomain $\Omega_{\bullet}^{(\alpha)}$.

with $w_{1_{\bullet}}^{(\alpha)}$ and $w_{2 \cdot}^{(\alpha)}=0,1,2, \ldots$ The dimensions $L_{x_{\bullet}}^{(\alpha)}$ and $L_{y_{\bullet}}^{(\alpha)}$ reppesent the dimensions of the (smallest) bounding rectangle, circumscribing the considered subdomain $\Omega_{\bullet}^{(\alpha)}$, as shown in Figure C.24.

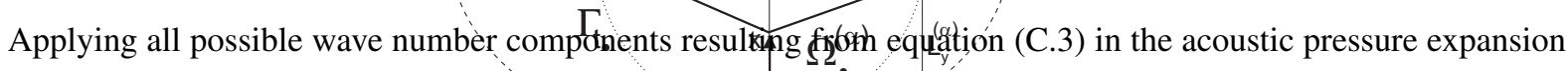
(7) leads to an infinite number of wave functions. In order to entwed the WBM in a numerical scheme, the number of wave functions for subdomain $\Omega_{\bullet}^{(\alpha)}$ is truncated to $n_{\boldsymbol{\bullet}, w}^{(\alpha)}$ functions. The adopted truncation rule is frequency dependent and selects the maximum values for the integers $w_{1}^{(\alpha)}$ and $w_{2 .}^{(\alpha)}$ such that the corresponding wave number $\frac{w_{s, m a x}^{(\alpha)} \pi}{L_{*}^{(\alpha)}}$ of the highest oscillating wave function of set $*$ is approximately equal to a user-defined truncation factor $N$ times the physical wavenumber $k_{\bullet}$ of the considered problem $[30,37]$. The rule is applied to all bounded subdomains such that the approximation field in adjacent subdomains exhibits a similar spatial variation along the interface.

\section{Appendix C.2. Wave functions for an unbounded subdomain}

The wave functions for the unbounded domains are chosen to explicitly comply with not only the Helmholtz equation 1, but also with the Sommerfeld radiation condition (3) at $\Gamma_{\infty}$. The following wave function set for unbounded domains exterior to a circular truncation curve with radius $R_{t_{\bullet}}$ (see Figure 2) is derived [39, 37], distinguishing between a c- and an s-set:

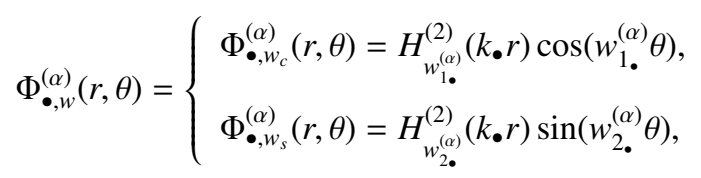

with $r$ and $\theta$ polar coordinates as defined in Figure C.25 $w_{1_{\bullet}}^{(\alpha)}=0,1,2, \ldots$ and $w_{2 \cdot}^{(\alpha)}=1,2,3, \ldots$ and $H_{n}^{(2)}(\bullet)$ is the $n$-th order Hankel function of the second kind. As for bounded domains, the series of functions (C.4) needs to truncated in order to be used in a numerical scheme. A similar truncation rule as for the bounded domains is used and determines the highest orders $w_{1_{\text {, } \max }}$ and $w_{2, \max }$ of the Hankel functions used in the exterior wave function expansion [39].

\section{Appendix C.3. Particular solution}

For unbounded acoustic problems a commonly used excitation for scattering problems is a plane wave source. For this source, the particular term yields:

$$
\hat{p}_{a, q}(\mathbf{r})=A e^{-\mathbf{j} \mathbf{k}_{\mathbf{a}} \cdot \mathbf{r}}
$$




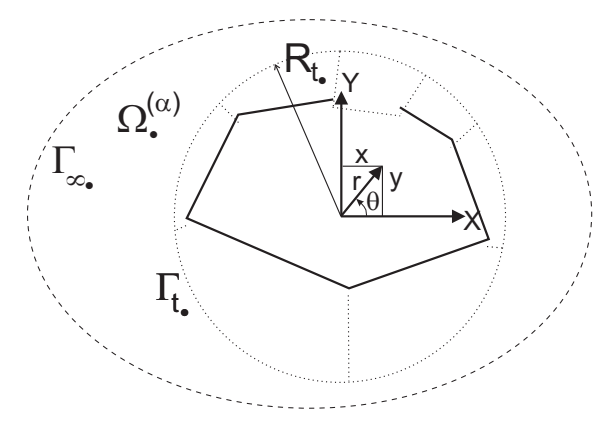

Figure C.25: Illustration of the coordinate system of an unbounded subdomain $\Omega_{\bullet}^{(\alpha)}$.

with $A$ the plane wave amplitude, $\mathbf{k}_{\mathbf{a}}=\left(k_{a x}, k_{a y}\right)=\left(k_{a} \cos \theta, k_{a} \sin \theta\right)$ the wave vector and $\theta$ the propagation angle, see also Figure 1.

\section{Appendix D. WBM system matrices for a unit cell transmission case}

The resulting WBM system matrix for a transmission case has the structure, given in equation (D.1).

The two first lines result from the two semi-unbounded acoustic domains. On the interfaces $\Gamma_{I_{a e}}$ the acoustic wave functions couple to the compound pressure field $p_{a}^{\left(1^{\prime}\right)}$, leading to non-zero coupling matrices $C_{a e}^{(1, *)}$, with $*=1,2$, for the associated bounded and unbounded wave functions of the involved bounded and unbounded levels. There is no direct coupling between both acoustic domains, nor a coupling between the acoustic and the bounded subdomains of the unbounded levels, describing the pressure field in the convex subdomains within the truncation circles. Only for the first acoustic subdomain a non-zero right hand side can be found, resulting from the particular solution of the incoming plane wave.

The third line in the system of equations gives the equations for the subdomain of the bounded level. Following the same reasoning as above, non-zero coupling matrices to the acoustic domains and the unbounded subdomains are seen. Again, there is no direct coupling between the wave functions of the bounded level and the bounded wave functions of the bounded subdomains $\Omega_{e}^{(1, \beta, \gamma)}$ of the unbounded levels. The non-zero right hand side results from the coupling with the acoustic domain and the particular solution of the incoming plane wave.

The following block of matrices gives the equations for the unbounded subdomains of the unbounded levels. The unbounded wave functions are only applied as weighting functions on the truncation circles they are associated to, resulting in coupling matrices between the wave functions of the bounded level and these of the other unbounded levels. In case an interface $\Gamma_{t, I_{e e}}^{\left(1^{\prime},(1, \beta, \gamma)\right)}$ exists between the compound subdomain $\Omega^{\left(1^{\prime}\right)}$ and $\gamma$-th bounded subdomain

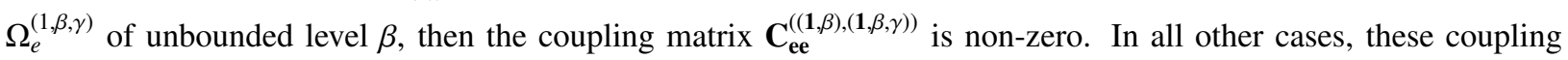
matrices are zero. For the given problem setting, the right hand sides are zero.

The final block of matrices results from the weighted residual approach for the bounded subdomains originating from the unbounded levels. These wave functions are only applied as weighting functions on their subdomain 
boundaries and interfaces. Consequently, non-zero coupling matrices are only obtained with the wave functions of the bounded level and the unbounded wave functions of the unbounded levels if a part of the truncation circles $\Gamma_{t, I_{e e}}^{(1, \beta)}$ belongs to the boundary of that subdomain $\Omega^{(1, \beta, \gamma)}$. If two subdomains $\Omega_{e}^{(1, \beta, \gamma)}$ and $\Omega_{e}^{(1, \beta, \chi)}$ of an unbounded level $\beta$ are adjacent, a non-zero coupling matrix $\mathbf{C}^{\left((1, \beta, \gamma),\left(1, \beta, \chi_{\mathrm{ee}}\right)\right)}$ is obtained. For the unit cell of Figure 6 , these coupling matrices are zero as there are no interfaces between the bounded subdomains in the first unbounded level. As only rigid boundaries and interfaces are considered, the right hand side of these equations is a zero vector. 


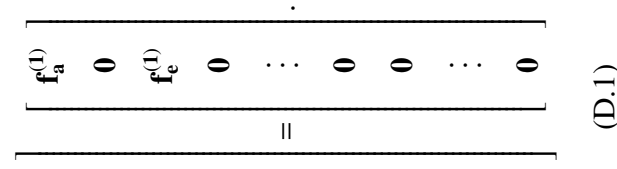

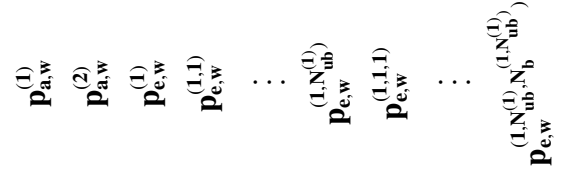

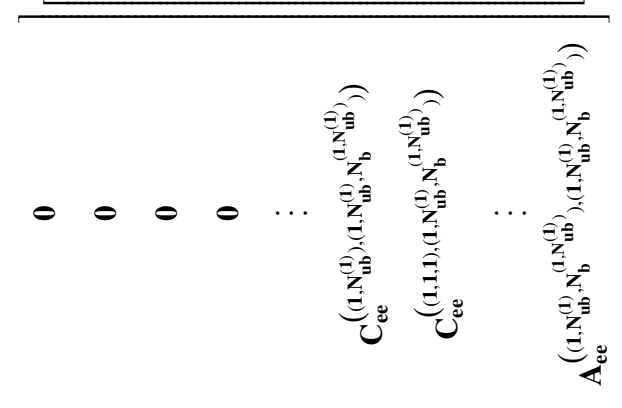

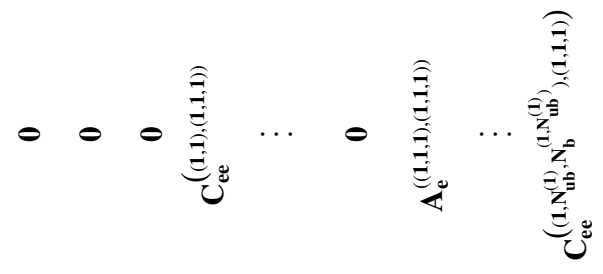

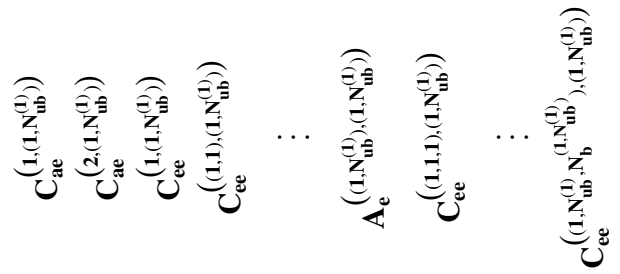

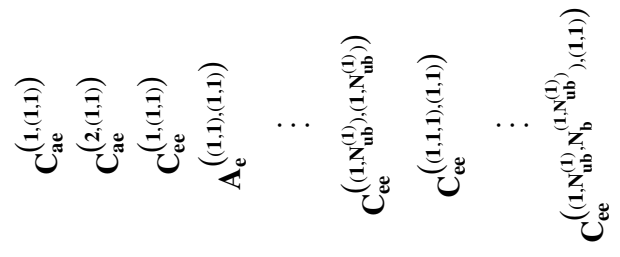

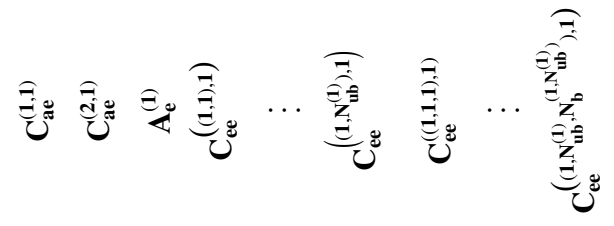

- बูy

$\theta_{i}$ e 
[1] R. Panneton and N. Atalla. Numerical prediction of sound tranmission through finite multilayer systems with poroelastic materials. J. Acoust. Soc. Am., 100:346-354, 1996.

[2] J.S. Lee, E.I. Kim, Y.Y. Kim, J.S. Kim, and Y.J. Kang. Optimal poroelastic layer sequencing for sound transmission loss maximization by topology optimization method. J. Acoust. Soc. Am., 122:2097-2106, 2007.

[3] E. Lind Nordgren, P. Göransson, J.-F. Deü, and O. Dazel. Vibroacoustic response sensitivity due to relative alignment of two anisotropic poro-elastic layers. JASA Express Letters, 133:426-430, 2013.

[4] C. Boutin, P. Royer, and J.L. Auriault. Acoustic absorption of porous surfacing with dual porosity. International Journal of Solids and Structures, 35:4709-4737, 1998.

[5] F.C. Sgard, X. Olny, N. Atalla, and F. Castel. On the use of perforations to improve the sound absorption of porous materials. Applied Acoustics, 66:625-651, 2005.

[6] E. Gourdon and M. Seppi. On the use of porous inclusions to improve the acoustical response of porous material: Analytical model and experimental verification. Applied Acoustics, 71:283-298, 2010.

[7] J.-P. Groby, A. Wirgin, and E. Ogam. Acoustic response of a periodic distribution of macroscopic inclusions within a rigid frame porous plate. Waves in Random and Complex Media, 18:409-433, 2008.

[8] J.-P. Groby, A. Duclos, O. Dazel, L. Boeckx, and L. Kelders. Enhancing absorption coefficient of a backed rigid frame porous layer by embedding circular periodic inclusions. J. Acoust. Soc. Am., 130:3771-3780, 2011.

[9] J.-P. Groby, C. Lagarrigue, B. Brouard, O. Dazel, and V. Tournat. Using simple shape three-dimensional rigid inclusions to enhance porous layer absorption. J. Acoust. Soc. Am., 136:1139-1148, 2014.

[10] C. Lagarrigue, J.-P. Groby, V. Tournat, O. Dazel, and O. Umnova. Absorption of sound by porous layers with embedded periodic arrays of resonant inclusions. J. Acoust. Soc. Am., 134:4670-4680, 2013.

[11] C. Boutin and F.-X. Bécot. Theory and experiments on poro-acoustics with inner resonators. Wave Motion, 54:76-99, 2015.

[12] J.-P. Groby, C. Lagarrigue, B. Brouard, O. Dazel, V. Tournat, and B. Nennig. Enhancing the absorption properties of acoustic porous plates by periodically embedding Helmholtz resonators. J. Acoust. Soc. Am., 137:273-280, 2015.

[13] J.-P. Groby, W. Lauriks, and T. Vigran. Total absorption peak by use of a rigid frame porous layer backed with a rigid multi-irregularities grating. J. Acoust. Soc. Am., 127:2865-2874, 2010.

[14] D. L. Johnson, J. Koplik, and R. Dashen. Theory of dynamic permeability and tortuosity in fluid-saturated porous media. J. Fluid Mech., $176: 379-402,1987$

[15] Y. Champoux and J.F. Allard. Dynamic tortuosity and bulk modulus in air-saturated porous media. Journal of Applied Physics, 70:1975-1979, 1991.

[16] J.F. Allard and N. Atalla. Propagation of Sound in Porous Media: Modeling Sound Absorbing Materials. John Wiley \& Sons, West Sussex, United Kingdom, 2nd edition, 2009.

[17] B. Nennig, Y. Renou, J.-P. Groby, and Y. Aurégan. A mode matching approach for modeling two dimensional porous grating with infinitely rigid or soft inclusions. J. Acoust. Soc. Am., 131:3841-3852, 2012.

[18] W. Desmet. A wave based prediction technique for coupled vibro-acoustic analysis. KULeuven, division PMA, PhD. thesis 98D12, 1998.

[19] E. Deckers, O. Atak, L. Coox, R. D’Amico, H. Devriendt, S. Jonckheere, K. Koo, B. Pluymers, D. Vandepitte, and W. Desmet. The Wave Based Method: an overview of 15 years of research. Wave Motion, 51:550-565, 2014

[20] E. Trefftz. Ein Gegenstück zum Ritzschen Verfahren. In Proceedings of the $2^{\text {nd }}$ International Congress on Applied Mechanics, Zurich, Switzerland, pages 131-137, 1926.

[21] B. Van Genechten, O. Atak, B. Bergen, E. Deckers, S. Jonckheere, J.S. Lee, A. Maressa, K. Vergote, B. Pluymers, D. Vandepitte, and W. Desmet. An efficient Wave Based Method for solving Helmholtz problems in three-dimensional bounded domains. Engineering Analysis with Boundary Elements, 36:63-75, 2012.

[22] B. Bergen, B. Van Genechten, D. Vandepitte, and W. Desmet. An efficient Trefftz-based method for three-dimensional Helmholtz problems in unbounded domains. Computer Modeling in Engineering E Sciences, 61(2):155-175, 2010. 
[23] C. Vanmaele, D. Vandepitte, and W. Desmet. An efficient wave based prediction technique for plate bending vibrations. Comput. Methods Appl. Mech. Engrg., 196:3178-3189, 2007.

[24] S. Jonckheere, D. Vandepitte, and W. Desmet. A Wave Based approach for the dynamic bending analysis of Kirchhoff plates under distributed deterministic and random excitation. Comput. Struct., 156:42-57, 2015.

[25] C. Vanmaele, K. Vergote, D. Vandepitte, and W. Desmet. Simulation of in-plane vibrations of 2D structural solids with singularities using an efficient wave based prediction technique. Computer Assisted Mechanics and Engineering Sciences, 19:135-171, 2012.

[26] E. Deckers, N.-E. Hörlin, D. Vandepitte, and W. Desmet. A Wave Based Method for the efficient solution of the 2D poroelastic Biot equations. Comput. Methods Appl. Mech. Engrg., 201-204:245-262, 2012.

[27] E. Deckers, B. Van Genechten, D. Vandepitte, and W. Desmet. Efficient treatment of stress singularities in poroelastic wave based models using special purpose enrichment functions. Comput. Struct., 89:1117-1130, 2011.

[28] B. Van Genechten and D. Vandepitte and W. Desmet. A direct hybrid finite element - Wave based modelling technique for efficient coupled vibro-acoustic analysis. Comput. Methods Appl. Mech. Engrg., 200:742-764, 2011.

[29] B. Van Genechten, B. Bergen, D. Vandepitte, and W. Desmet. A Trefftz-based numerical modelling framework for Helmholtz problems with complex multiple scatterer configurations. Journal of Computational Physics, 229(18):6623-6643, 2010.

[30] B. Van Genechten, K. Vergote, D. Vandepitte, and W. Desmet. A Multi-Level Wave Based numerical modelling framework for the steadystate dynamic analysis of bounded Helmholtz problems with multiple inclusions. Comput. Methods Appl. Mech. Engrg., 199:1881-1905, 2010.

[31] E. Deckers, S. Jonckheere, D. Vandepitte, and W. Desmet. Modelling Techniques for Vibro-Acoustic Dynamics of Poroelastic Materials. Arch. Computat. Methods Eng., 22:183-236, 2015.

[32] F. Bloch. Über die Quantenmechanik der Elektronen in Kristallgittern. Zeitschrift für Physik A Hadrons and Nuclei, 52:555-600, 1929.

[33] L. Brillouin. Wave propagation in periodic structures. McGraw-Hill Book Company, 2nd edition, 1946.

[34] D. Colton and R. Kress. Inverse acoustic and electromagnetic scattering theory. Springer-Verlag, Berlin, Heidelbert, New York, 2nd edition, 1998.

[35] E. Deckers, B. Bergen, B. Van Genechten, D. Vandepitte, and W. Desmet. An efficient Wave Based Method for 2D acoustic problems containing corner singularities. Computer Methods in Applied Mechanics and Engineering, 241-244:286-301, 2012.

[36] B. Pluymers, B. Van Hal, D. Vandepitte, and W. Desmet. Trefftz-based methods for time-harmonic acoustics. Archives of Computational Methods in Engineering, 14(4):343-381, 2007.

[37] B. Pluymers. Wave based modelling methods for steady-state vibro-acoustics. KU Leuven, division PMA, PhD. thesis 2006D04, Leuven, 2006.

[38] M.J. Grote and J.B. Keller. On nonreflecting Boundary Conditions. J. Comp. Phys., 122:231-243, 1995.

[39] B. Bergen, B. Pluymers, B. Van Genechten, D. Vandepitte, and W. Desmet. A Trefftz based method for solving Helmholtz problems in semi-infinite domains. Engineering Analysis with Boundary Elements, 36:30-38, 2012.

[40] O. Atak, S. Jonckheere, E. Deckers, D. Huybrechs, B. Pluymers, and W. Desmet. A hybrid Boundary Element-Wave Based Method for the efficient solution of bounded acoustic problems with inclusions. Comput. Methods Appl. Mech. Engrg., 283:1260-1277, 2015.

[41] O. Atak. Wave Based Modeling Methods for Acoustic Inclusion and Multiple Scattering problems in the Mid-Frequency Range. KU Leuven, division PMA, PhD. thesis, 2014.

[42] D. Lafarge, P. Lemarinier, J.F. Allard, and V. Tarnow. Dynamic compressibility of air in porous structures at audible frequencies. Journal of the Acoustical Society of America, 102:1995-2006, 1997.

[43] J.D. Benamou and B. Depres. A domain decomposition method for the Helmholtz equation and related optimal control problems. J. Comp. Phys., 136:68-82, 1997. 